Review Article

\title{
The Effectiveness and Safety of Moxibustion for Treating Knee Osteoarthritis: A PRISMA Compliant Systematic Review and Meta-Analysis of Randomized Controlled Trials
}

\author{
Ting Yuan $\mathbb{D},{ }^{1}$ Jun Xiong $\mathbb{D},{ }^{2}$ Xue Wang $\mathbb{D},{ }^{1}$ Jun Yang $\mathbb{D},{ }^{1}$ Yunfeng Jiang $\mathbb{D},{ }^{2}$ \\ Xiaohong Zhou $\mathbb{D}^{2},{ }^{2}$ Kai Liao $\mathbb{D}^{2},{ }^{2}$ and Lingling $\mathrm{Xu} \mathbb{D}^{2}$ \\ ${ }^{1}$ Jiangxi University of Traditional Chinese Medicine, Nanchang, China \\ ${ }^{2}$ The Affiliated Hospital of Jiangxi University of Traditional Chinese Medicine, Nanchang, China \\ Correspondence should be addressed to Jun Xiong; xiongjun196071@163.com
}

Received 25 July 2019; Revised 12 November 2019; Accepted 27 November 2019; Published 18 December 2019

Guest Editor: Fang Zeng

Copyright (c) 2019 Ting Yuan et al. This is an open access article distributed under the Creative Commons Attribution License, which permits unrestricted use, distribution, and reproduction in any medium, provided the original work is properly cited.

\begin{abstract}
Background. Knee osteoarthritis (KOA) seriously affects people's life. Therefore, it has already become a worldwide health concern. Moxibustion has a significant clinical effect on KOA. This systematic review and meta-analysis is performed to renew previous studies and strictly evaluate the quality of RCT and thus test the effect and safety of moxibustion for KOA. Objective. To evaluate the effectiveness and safety of moxibustion treatment for alleviating pain and improving lower limb function for patients with KOA. Materials and Methods. CNKI (1979 2019), CBM (1979 2019), VIP (1989 2019), WF (1998 2019), PubMed (1966 2019), Embase (1980 2019), Cochrane Library, and Web of Science (1900 2019) were all retrieved by a computer from their inception to June 02,2019 , replenished by manual retrieval of relevant bibliographies. Randomized controlled trials (RCTs) were included if moxibustion was compared to western medicine or negative control (placebo moxibustion or no treatment or UC) for treating KOA. The primary outcomes were the total effect and the Western Ontario and McMaster Universities Osteoarthritis Index (WOMAC scale). The secondary outcomes include VAS, Symptom score, Lysholm score, and Lequesne score. RCTs were collected, and the quality of evidence was evaluated by using the Jadad scale and Cochrane risk assessment tools. We used RevMan5.3.0 software for meta-analysis. Results. A total of 39 RCTs were included, including 3293 patients. In the assessment of the quality, the evidence differs from low to high based on the Cochrane Bias Evaluation Tools and Jadad scale. Fourteen trials were of high quality, ten were of moderate quality, and 15 were of low quality. Therefore, the quality of the included studies was moderate. In this study, there were $66.67 \%$ of the literature, and only $17.95 \%$ of the literature correctly reported randomized grouping and allocation of hidden information, respectively. In adverse reactions, only 13 trials included were reported in the study. The main adverse reactions of moxibustion are burns and blisters, whereas the western medicine group was in epigastric discomfort. As for the total effective rate, the meta-analysis of 27 RCTs showed a significant effect of moxibustion VS western medicine $\left(\mathrm{RR}=1.20,95 \% \mathrm{CI}=1.16\right.$ to $\left.1.25, I^{2}=45 \%, P=0.007\right)$; as for the WOMAC scale, the subgroup meta-analysis of 13 trials showed that there was a statistically significant effect of moxibustion VS western medicine $(\mathrm{MD}=-11.08,95 \% \mathrm{CI}=-11.72$ to $\left.-10.44, I^{2}=98 \%, P<0.00001\right)$ and 2 trials on moxibustion VS negative control $\left(\mathrm{MD}=-8.38,95 \% \mathrm{CI}=-12.69\right.$ to $-4.06, I^{2}=0 \%$, $P=0.77)$; as for the VAS score, the meta-analysis of 6 trials showed that there was a significant effect of moxibustion VS western medicine $\left(\mathrm{MD}=-2.12,95 \% \mathrm{CI}=-2.30\right.$ to $\left.-1.93, I^{2}=98 \%, P<0.00001\right)$; as for the symptom score, the meta-analysis of 7 trials showed that there was a significant effect of moxibustion VS western medicine $\left(\mathrm{MD}=-0.81,95 \% \mathrm{CI}=-1.24\right.$ to $-0.37, I^{2}=50 \%$, $P=0.06$ ); as for the Lysholm score, the meta-analysis of 5 trials showed that there was a significant effect of moxibustion VS western medicine ( $\mathrm{MD}=7.61,95 \% \mathrm{CI}=6.04$ to $\left.9.17, I^{2}=95 \%, P<0.00001\right)$; and as for the Lequesne score, the meta-analysis of 3 trials showed that there was a significant effect of moxibustion VS western medicine (MD $=3.29,95 \% \mathrm{CI}=2.93$ to $3.65, I^{2}=99 \%$, $P<0.00001$ ). Conclusion. Moxibustion treatment for KOA is more effective than the positive control (western medicine) or negative control (placebo moxibustion or no treatment or UC), and there were fewer adverse reactions to moxibustion. Due to the universally low quality of the eligible trials, it still needs further large-scale and high-quality randomized controlled trials to verify the effectiveness and safety of moxibustion in the treatment of KOA.
\end{abstract}




\section{Introduction}

Knee osteoarthritis (KOA) is the most usual and frequent disease of arthritis caused by subchondral bone hyperplasia, which destroys joints and generates deformations increasingly, affecting the functions of knee joints severely, also named as proliferative osteoarthritis or degenerative arthritis, or hypertrophic arthritis $[1,2]$. The prominent clinical features of KOA are pain, stiffness, swelling, joint cavity effusion, and motor dysfunction [2]. The etiology of KOA is varied, mainly caused by meniscus injury. The meniscus is composed of fibrous cartilage, one inside and one outside, and is located in the joint space of the knee, which acts as a buffer against shock and cartilage protector [3]. KOA is a primary reason for pain and functional limitation, which reduces the patients' quality of life (QOL) significantly [4]. In China, KOA mainly occurs in middleaged and old people, with the symptomatic prevalence of $10.3 \%$ and $5.7 \%$, respectively [5]. KOA and hip OA constitute the major global health burden together, ranking 11th topmost among global contributors to disability and 38th topmost in disability-adjusted life years [6]. Today, although most guidelines recommend the multimodality drug and nondrug methods as the treatment for KOA [7], long-term use can cause significant adverse reactions, such as gastrointestinal irritation and bleeding, perforated ulcers, hepatic toxicity, and renal toxicity. Hence, nonpharmacological therapy is frequently used in China, including complementary and alternative medicines, for instance, of moxibustion [8].

Moxibustion, a traditional Chinese medicine (TCM) treatment, is made of moxibustion material, mainly moxibustion leaves, produces heat to stimulate specific acupuncture points or parts of the body surface, regulates the function of visceral organs by stimulating meridian qi, and to achieve the purpose of treating diseases [9]. And it is usually used in patients with KOA for its representative function of nondrug intervention in TCM [10]. Although there are many clinical trials and studies on moxibustion in the treatment of KOA, systematic review and metaanalysis of moxibustion or moxibustion combined with western medicine in the treatment of KOA is still insufficient, and a lot of clinical studies have confirmed that western medicine has obvious side effects and is not conducive to long-term use. Moxibustion has fewer side effects and higher safety. Therefore, this study compared the effectiveness and safety of moxibustion in the treatment of KOA in accordance with the method of systematic review and meta-analysis.

Presently, there were six systematic reviews and metaanalysis of moxibustion for KOA [11-16]. Among them, there were four Chinese articles [11-14] and two $[15,16]$ English articles. The authors of five articles were from China and one from South Korea. Two articles in Chinese literature compared the effectiveness of moxibustion and other therapies for KOA $[11,12]$. Although there were two papers studying the effectiveness of moxibustion in the treatment of $\mathrm{KOA}$, there are also some disadvantages, for instance, too few included samples, low quality, or long publication time $[13,14]$. Although two of the English articles also studied the effectiveness of moxibustion for $\operatorname{KOA}[15,16]$, only one searched the English database [15], and the other had deficiencies such as too little sample size and few outcome indicators [16]. Hence, the aim of this study is to renew, improve, and strictly evaluate the quality of RCTs to test the effectiveness and safety of moxibustion in the treatment of KOA and better guide the clinical practice of acupuncture and moxibustion.

\section{Methods}

2.1. Protocol Register. We conducted this systematic review and meta-analysis strictly according to the PRISMA (The Preferred Reporting Items for Systematic Review and Meta-analysis) statement [17] (S1 PRISMA Checklist). Moreover, we published this protocol in PROSPERO 2015 CRD42015016920 in advance. It is available from http:// www.crd.york.ac.uk/PROSPERO/display_record.php?ID= CRD42015016920.

2.2. Types of Studies. Only randomized clinical trials (RCTs) were included in this systematic review and metaanalysis. There was no limitation on patients' age, gender, course of the disease, syndrome type, and source of cases. The study subjects had recognized diagnostic criteria and therapeutic effectiveness criteria, and they were all diagnosed with KOA. The commonly used diagnostic criteria in China followed the guidelines for diagnosis and treatment of osteoarthritis, guidelines for clinical research of new traditional Chinese medicine, and criteria for diagnosis and therapeutic effectiveness of TCM diseases and syndromes revised by the orthopedic branch of the Chinese medical association in 2007. The diagnostic criteria of knee arthritis of the American College of Rheumatology (ACR) were followed abroad. There were no language restrictions.

2.3. Exclusion Criteria. Duplicate detection and publications, non-moxibustion intervention studies, expert experience, case report, theoretical studies, experimental studies, unclear diagnostic criteria, abstract and conference articles, meaningless interventions in the control group, and incomplete data of the results will be excluded.

2.4. Types of Interventions. We included the studies that used various forms of moxibustion (e.g., direct or indirect moxibustion, heat-sensitive moxibustion, gingpao moxibustion, warm needling, or salt-separated moxibustion) as the single therapy or as the main part of a combination treatment with other interventions (e.g., western medicine). The trials, whether the control group received the consistent concomitant treatments as the treatment group, would also be included. And the trials that moxibustion was used as an ancillary treatment would be excluded. 
2.5. Types of Comparators. We included comparators of no treatment, placebo moxibustion, or related standard treatment for KOA, including western medicine and conventional therapies. If the design of the trial does not consider the evaluation of the effectiveness of moxibustion, the trial will be excluded (e.g., the control group was treated with unproven efficacy therapy, or two different forms of moxibustion were compared) or if they adopted comparators between treatments whose expected effectiveness was consistent to that of moxibustion (e.g., acupuncture).

\subsection{Types of Outcome Measures}

2.6.1. Major Outcomes. The primary outcomes included the total effect and the WOMAC scale. According to the total WOMAC score of the patient, the effectiveness was evaluated concerning the Nimodipine method, namely, index improvement rate $=[$ (pretreatment symptom score-posttreatment symptom score $) \div$ pretreatment symptom score $] \times 100 \%$. Cure: index improvement rate $>75 \%$; remarkable effect: index improvement rate $\geq 50 \%$ and $\leq 75 \%$; effective: index improvement rate $\geq 30 \%$ and $<50 \%$; invalid: index improvement rate $<30 \%$. The WOMAC scale was reliable reported pain, stiffness, and function measures for osteoarthritis by the global scale value of the Western Ontario and McMaster Universities Osteoarthritis Index (WOMAC) questionnaire. Pain and function scores were converted to a 0-100 scale using the WOMAC items that assess pain (five items) and function (17 items). Higher scores on the WOMAC indicate worse pain and functional status.

2.6.2. Additional Outcome(s). The secondary outcomes included VAS, Symptom score, Lysholm score, Lequesne score, and adverse reactions: Visual Analogue scale (VAS) (score range, 0 10): higher scores indicate worse knee pain; Symptom score: according to the guidelines for clinical research on new Chinese medicine in 2002 on the grading of osteoarthritis symptoms, according to the pain, joint swelling, joint activity, morning stiffness, and TCM syndrome diagnosis criteria for grading; Lysholm score: mainly used to evaluate knee flexion and extension activity, consisting of 8 questions with a score of $0 \sim 100$. Score above 95 points is excellent, $94 \sim 85$ is good, $84 \sim 65$ is fair, and less than 65 is poor; Lequesne score: the knee osteoarthritis severity index score scale was used to evaluate the severity of knee osteoarthritis in patients from the aspects of joint motion pain, night pain, morning stiffness, and daily activities. The score on the score scale was directly proportional to the severity of symptoms; and adverse reactions: the severity, frequency, and duration were observed.

2.7. Information Sources. We adopted a comprehensive and exhaustive search strategy, including searching electronic databases, manually searching references, contacting pharmaceutical companies, and lead authors. We searched the following electronic databases: CNKI, CBM, VIP, WF,
PubMed, Embase, Web of Science, and the Cochrane Library from their inception to June 2, 2019, without language limitation. On July 2, we conducted a repeat search with the same search strategy to fill in the gaps. We also manually searched the relevant journals and bibliographies. There were no limitations on publication years or publication status.

2.8. Search Strategy. The comprehensive search strategy for PubMed is listed in Table 1.

2.9. Data Extraction. Firstly, according to the PICOST principle, the standard data extraction table was set up in advance. Before the formal data extraction, two preliminary tests were carried out to ensure the quality of data extraction. Then, two evaluators (Ting Yuan and Jun Yang) read the titles and abstracts back-to-back independently to conduct a preliminary screening of the literature and then read the full text in the same way to determine the final included studies. Whenever possible, an intention-to-treat (ITT) analysis of the missing data was performed. Relevant details included author information, year, sample size, a period of treatment, diagnostic criteria, outcome measures, interventions, comparators, and the lost follow-up situation and then crosschecked the results of the included trials. If there is any difference, the inclusion will be decided by discussion or the third evaluator (Xue Wang). When there is incomplete information in the study, it is necessary to contact the first author to obtain relevant data according to the provisions of the standardized protocol.

2.10. Quality Assessment. The evaluation criteria of the Jadad scale were strictly followed [18], and the Cochrane risk assessment tool [19] was used to evaluate the included studies. According to Cochrane Handbook 5.2.0, quality reviews and risk of bias are available. The details are as follows: random sequence generation, allocation concealment, blinding of participants and doctors, blinding of outcome evaluator, incomplete outcome data, selective reporting, and other bias. Each item was classified according to a high, low, or unclear risk of bias that is represented as high $(\mathrm{H})$, low $(\mathrm{L})$, and unclear $(\mathrm{U})$, respectively. The quality evaluation results included in the test were cross-examined by two evaluators (Ting Yuan and Jun Yang), and any differences that were difficult to determine could be solved by discussion or the third evaluator (Xue Wang).

\subsection{Summary Measures and Data Synthesis.} Meta-analysis was performed with RevMan5.3.0 software. The heterogeneity test was conducted between studies, with $P<0.1$ and $I^{2}>50 \%$ as the test level. When there was no statistical heterogeneity between studies, the fixed effect model was adopted. In the absence of clinical or methodological heterogeneity, a randomized effect model was used. Continuous variables used mean difference $\mathrm{MD}$; relative ratio (RR) was adopted as the categorical variable, and both effect sizes were expressed as 95\% CI. $P \leq 0.05$ was 
TABLE 1: Search strategy.

\begin{tabular}{|c|c|}
\hline \multicolumn{2}{|c|}{ Source: PubMed; searched on June 12, 2019} \\
\hline Search & Query \\
\hline$\# 1$ & $\begin{array}{c}\text { "knee osteoarthritis" [Ti/Ab] or "knee pain" [Ti/Ab] } \\
\text { or "osteoarthritis of knee" [Ti/Ab] or "knee joint } \\
\text { osteoarthritis" [Ti/Ab] or "knee arthritis" [Ti/Ab] or } \\
\text { "knee gonitis" [Ti/Ab] }\end{array}$ \\
\hline \#2 & "moxibustion" [title/abstract] \\
\hline \#3 & $\begin{array}{c}\text { "randomized controlled trial" }[\mathrm{Ti} / \mathrm{Ab}] \text { or "clinical } \\
\text { trial" }[\mathrm{Ti} / \mathrm{Ab}]\end{array}$ \\
\hline \#4 & $\# 1$ and $\# 2$ and \#3 \\
\hline \#5 & "knee osteoarthritis" [MeSH] \\
\hline \#6 & “moxibustion" [MeSH] \\
\hline$\# 7$ & $\begin{array}{c}\text { "randomized controlled trial" }[\mathrm{MeSH}] \text { or "controlled } \\
\text { clinical trial" }[\mathrm{MeSH}]\end{array}$ \\
\hline$\# 8$ & $\# 5$ and $\# 6$ and \#7 \\
\hline \#9 & $\# 4$ or \#8 \\
\hline
\end{tabular}

considered statistically significant. If there was significant clinical heterogeneity between studies, only descriptive analysis was performed. If necessary, sensitivity analysis is selected to test the stability of results, and RevMan5.3.0 software is used to analyze the publication bias.

2.12. Risk of Bias across Trials. If the RCTs were more than 10 , the funnel plot might be used to detect publication bias of the included trials in this meta-analysis.

2.13. Additional Analysis. Subgroup analysis and sensitivity analysis were performed to explore the potential heterogeneity and confounders on outcomes. And the subgroup analysis is predefined in the PROSPERO protocol.

2.14. Ethical Statement. There were no ethical approval requirements for this study.

\section{Results}

3.1. Search Results. A total of 1499 studies were retrieved at the initial search. NoteExpress 2.2.0 software was used for statistical management, and unqualified studies were excluded. At last, a total of 39 eligible trials were included, as shown in Figure 1.

3.2. Study Characteristics. The characteristics of all included RCTs are documented. All RCTs were published from 2006 to 2019. There were 1640 patients in the treatment group and 1653 in the control group, respectively. There were four RCTs with three groups, but only two of them met the inclusion and exclusion criteria. Therefore, we only extracted the baseline data of these two groups. The number of patients in each trial varied from 21 to 110 . A majority of the patients were in the outpatient or inpatient department. There were 22 RCTs using the ACR (American College of Rheumatology) diagnostic criteria, 8 using the guiding principles of clinical research on new drugs of traditional Chinese medicine and 9 using the guidelines for diagnosis and treatment of osteoarthritis 2007. Besides, the outcome data and other information of each included study are listed in Table 2.

3.2.1. Types of Studies. All of the eligible trials were randomized clinical trials (RCTs) and five were multicenter RCTs $[2,30,38,39,53]$.

3.2.2. Types of Intervention. Sixteen RCTs $[21-24,26,29,32$, $37,40-42,44,45,48,53]$ adopt warm-needling moxibustion treatment; ten RCTs $[2,25,30,31,38,39,49,51,55,57]$ adopt moxibustion; two RCTs [28, 50] adopt heat-sensitive moxibustion; two RCTs $[27,54]$ adopt thunder fire moxibustion; two RCTs [33, 52] adopt herb cake-partitioned moxibustion (HCPM); two RCTs [34, 56] adopt separated aconite cake mild moxibustion (SACM); two RCTs [46, 47] adopt crude herb moxibustion; one RCT [20] adopts threevolt heat-sensitive wheat moxibustion; one RCT [35] adopts gingpao moxibustion; one RCT [36] adopts salt-separated moxibustion; and one RCT [43] adopts Sanqi cake moxibustion.

3.2.3. Types of Control. Thirty-three RCTs [2, 20-25, 27-29, 32-37, 40-48, 50-57] adopt western medicine treatment; four RCTs $[30,31,39,49]$ adopt placebo moxibustion treatment; one RCTs [25] adopt no treatment, and one RCTs [38] adopt UC treatment.

3.2.4. Types of Outcome Measures. Twenty-seven RCTs $[2,20-25,28,29,32-37,40-42,44,45,48,50-55,57]$ assess the total effective rate. Fifteen RCTs $[2,22-27,33,35,36,38,40,42,47,53]$ used the WOMAC scale to assess the pain and physical function, six RCTs $[2,20,21,27,45,51]$ selected the VAS scale, seven RCTs $[21,35,45-47,50,56]$ selected the Symptom score, five RCTs $[21,22,32,45,51]$ selected the Lysholm score, and three RCTs $[23,25,55]$ selected the Lequesne score to assess pain or symptoms, respectively.

\subsection{Risk of Bias Assessment}

(1) Randomization: 16 RCTs [2, 20, 21, 28, 32, 33, 36, 42, $44,45,48,50,51,53,55,56]$ were randomized by the random number table, 6 RCTs $[26,30,38-40,49]$ were randomized by a computer, 4 RCTs $[22,35,46,47]$ were randomized by draw, and 13 RCTs [23-25, 27, 29, 31, 34, 37, 41, 43, 52, 54, 57] were randomized word only. (2) Allocation hiding: only 7 RCTs $[26,30,38-40,49,53]$ mentioned proper allocation hiding, 20 RCTs mentioned using random number table or draw random, and 13 RCTs did not mention whether allocation hiding. (3) Blind method: only 2 RCTs $[39,49]$ implemented the doubleblind method, 3 RCTs $[30,31,40]$ implemented the single-blind method, the rest of the experiments did not mention whether the blind method was used. (4) Selective report: all studies reported preset outcome indicators; (5) Follow-up and abscission: only 13 RCTs $[2,26,30,32,42,46,47,49,54,56]$ 


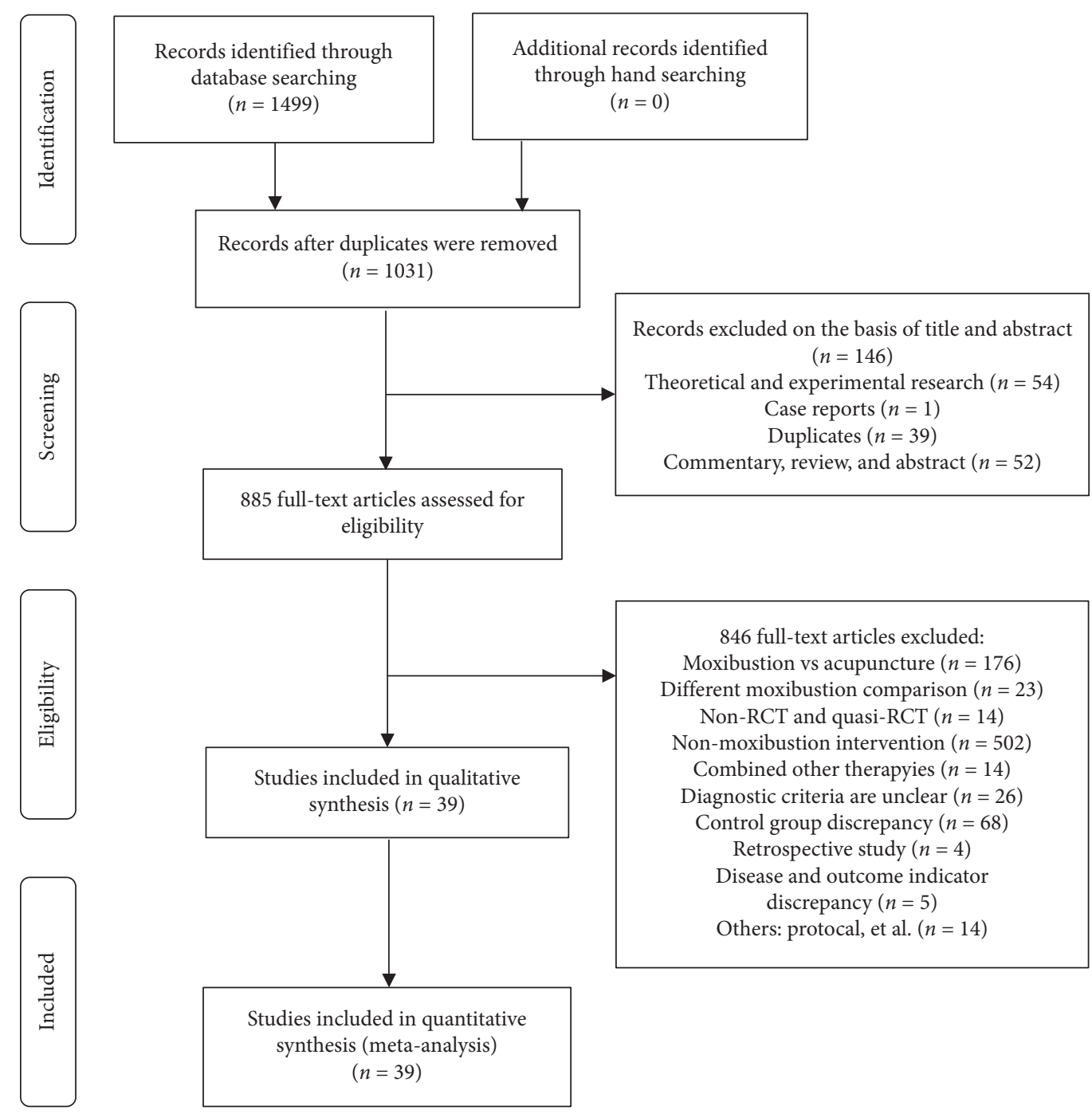

FIGURE 1: Flow diagram of the study.

reported the number of cases of abscission and its causes in detail, as shown in Table 3 and Figures 2 and 3.

\subsection{Quantitative Review and Meta-Analysis}

3.4.1. Total Effective Rate. The forest plot illustrating the results of the meta-analysis for the total effective rate is shown in Figure 4; twenty-seven RCTs compared the effectiveness of moxibustion versus western medicine alone and showed a significant effect of moxibustion on $\mathrm{KOA}\left(\mathrm{RR}=1.20,95 \% \mathrm{CI}=1.16\right.$ to $1.25, I^{2}=45 \%$, $P=0.007)$.

\subsubsection{WOMAC Scale}

(1) Moxibustion VS Western Medicine. The subgroup analysis showed that meta-analysis of the data on using the WOMAC scale (Figure 5), and eight RCTs compared the effectiveness of moxibustion versus western medicine alone and showed a significant effect of moxibustion on KOA
$\left(\mathrm{MD}=-11.08, \quad 95 \% \mathrm{CI}=-11.72\right.$ to $-10.44, \quad I^{2}=98 \%$, $P<0.00001)$.

(2) Moxibustion VS Negative Control. The subgroup analysis showed that 2 RCTs compared the effectiveness of moxibustion versus negative control and showed a significant effect of moxibustion on KOA $(\mathrm{MD}=-8.38, \quad 95 \%$ $\mathrm{CI}=-12.69$ to $\left.-4.06, I^{2}=0 \%, P=0.77\right)$.

3.4.3. VAS Score. The forest plot illustrating the results of the meta-analysis for the VAS score is shown in Figure 6. Six RCTs compared the effectiveness of moxibustion versus western medicine alone and showed a significant effect of moxibustion on $\mathrm{KOA}(\mathrm{MD}=-2.12,95 \% \mathrm{CI}=-2.30$ to -1.93 , $\left.I^{2}=98 \%, P<0.00001\right)$.

3.4.4. Symptom Score. The forest plot illustrating the results of the meta-analysis for the Symptom score is shown in Figure 7, and seven RCTs compared the effectiveness of moxibustion versus western medicine alone and showed a 
TABLE 2: Basic characteristics of eligible RCTs.

\begin{tabular}{|c|c|c|c|c|c|c|c|}
\hline \multirow{2}{*}{ Study ID } & \multirow{2}{*}{$\begin{array}{l}\text { Sample } \\
\text { size T/C }\end{array}$} & \multirow{2}{*}{$\begin{array}{l}\text { Diagnostic } \\
\text { criteria }\end{array}$} & \multicolumn{2}{|l|}{ Intervention } & \multirow{2}{*}{$\begin{array}{c}\text { Period } \\
(\mathrm{w})\end{array}$} & \multirow{2}{*}{ Outcome } & \multirow{2}{*}{$\begin{array}{c}\text { Drop } \\
\text { out }\end{array}$} \\
\hline & & & Trial group & Control group & & & \\
\hline Dai et al. [2] & $80(38 / 36)$ & 2 & Moxibustion & Celecoxib & $4 / 4$ & $\begin{array}{c}\text { Total effective rate, VAS, } \\
\text { WOMAC }\end{array}$ & $\begin{array}{l}\mathrm{T}: 2, \\
\mathrm{C}: 4\end{array}$ \\
\hline Bu et al. [20] & $62(31 / 31)$ & 2 & $\begin{array}{l}\text { Three-volt heat-sensitive } \\
\text { wheat moxibustion }\end{array}$ & $\begin{array}{l}\text { Futaline } \\
\text { emulsion }\end{array}$ & $4 / 4$ & Total effective rate, VAS & NR \\
\hline Yao [21] & $80(40 / 40)$ & ACR & $\begin{array}{l}\text { Warm-needling } \\
\text { moxibustion }\end{array}$ & Ibuprofen & $8 / 8$ & $\begin{array}{l}\text { Total effective rate, VAS, LKSS, } \\
\text { Symptom score }\end{array}$ & NR \\
\hline Chao [22] & $82(41 / 41)$ & ACR & $\begin{array}{l}\text { Warm-needling } \\
\text { moxibustion }\end{array}$ & Ibuprofen & $3 / 3$ & WOMAC, LKSS & NR \\
\hline Li et al. [23] & $80(40 / 40)$ & ACR & $\begin{array}{l}\text { Warm-needling } \\
\text { moxibustion }\end{array}$ & Ibuprofen & $2 / 2$ & $\begin{array}{c}\text { Total effective rate, WOMAC, } \\
\text { Lequesne-Mery }\end{array}$ & NR \\
\hline Ren [24] & $\begin{array}{c}108(54 / \\
54)\end{array}$ & ACR & $\begin{array}{l}\text { Warm-needling } \\
\text { moxibustion }\end{array}$ & $\begin{array}{l}\text { Glucosamine } \\
\text { sulfate tablets }\end{array}$ & $20 / 20$ & Total effective rate, WOMAC & NR \\
\hline $\begin{array}{l}\text { Chen et al. } \\
{[25]}\end{array}$ & $60(30 / 30)$ & 2 & moxibustion & $\begin{array}{l}\text { Diclofenac } \\
\text { sodium }\end{array}$ & $4 / 4$ & $\begin{array}{c}\text { Total effective rate, WOMAC, } \\
\text { Lequesne-Mery }\end{array}$ & NR \\
\hline $\begin{array}{l}\text { Wang et al. } \\
{[26]}\end{array}$ & $50(25 / 21)$ & ACR & $\begin{array}{l}\text { Warm-needling } \\
\text { moxibustion }\end{array}$ & No treatment & $3 / 3$ & Total effective rate, WOMAC & $\begin{array}{l}\mathrm{T}: 0 \\
\mathrm{C}: 4\end{array}$ \\
\hline $\begin{array}{l}\text { Huang and } \\
\text { Ji [27] }\end{array}$ & $60(30 / 30)$ & 2 & Thunder fire moxibustion & Celecoxib & $5 / 5$ & WOMAC, VAS & NR \\
\hline Chi et al. & $\begin{array}{l}120(40 / \\
40 / 40)\end{array}$ & 1 & Heat-sensitive moxibustion & $\begin{array}{c}\text { Sodium } \\
\text { hyaluronate }\end{array}$ & $4 / 4$ & Total effective rate & NR \\
\hline Ji [29] & $96(48 / 48)$ & ACR & $\begin{array}{l}\text { Warm-needling } \\
\text { moxibustion }\end{array}$ & $\begin{array}{l}\text { Nabumetone } \\
\text { capsules }\end{array}$ & $6 / 6$ & Total effective rate & NR \\
\hline $\begin{array}{l}\text { Ren et al. } \\
{[30]}\end{array}$ & $\begin{array}{l}150(69 / \\
67)\end{array}$ & ACR & Moxibustion & $\begin{array}{l}\text { Placebo } \\
\text { moxibustion }\end{array}$ & $6 / 6$ & Non & $\begin{array}{l}\mathrm{T}: 8 \\
\mathrm{C}: 6\end{array}$ \\
\hline Sit et al. [31] & $\begin{array}{l}110(55 / \\
55)\end{array}$ & ACR & Moxibustion & $\begin{array}{c}\text { Placebo } \\
\text { moxibustion }\end{array}$ & $6 / 6$ & WOMAC & NR \\
\hline Cui [32] & $\begin{array}{l}90(29 / 30 / \\
30)\end{array}$ & 2 & $\begin{array}{l}\text { Warm-needling } \\
\text { moxibustion }\end{array}$ & $\begin{array}{l}\text { Glucosamine } \\
\text { sulfate tablets }\end{array}$ & $3 / 3$ & $\begin{array}{c}\text { Total effective rate, WOMAC, } \\
\text { Lysholm }\end{array}$ & $\begin{array}{l}\mathrm{T}: 1 \\
\mathrm{C}: 0\end{array}$ \\
\hline $\begin{array}{l}\text { Huang et al. } \\
{[33]}\end{array}$ & $\begin{array}{c}120(60 / \\
60)\end{array}$ & 1 & HCPM & $\begin{array}{l}\text { Diclofenac } \\
\text { sodium }\end{array}$ & $4 / 4$ & Total effective rate, WOMAC & NR \\
\hline $\begin{array}{l}\text { Zhang and } \\
\mathrm{Li}[34]\end{array}$ & $60(30 / 30)$ & 1 & SACM & $\begin{array}{l}\text { Diclofenac } \\
\text { sodium }\end{array}$ & $2 / 2$ & Total effective rate & NR \\
\hline Wang [35] & $60(30 / 30)$ & 2 & Gingpao moxibustion & $\begin{array}{c}\text { Sodium } \\
\text { hyaluronate }\end{array}$ & $8 / 8$ & $\begin{array}{c}\text { Total effective rate, WOMAC, } \\
\text { VAS, Symptom score }\end{array}$ & NR \\
\hline $\begin{array}{l}\text { Deng et al. } \\
{[36]}\end{array}$ & $70(45 / 45)$ & 2 & Salt-separated moxibustion & Ibuprofen & $4 / 4$ & Total effective rate, WOMAC & NR \\
\hline Li et al. [37] & $80(42 / 38)$ & ACR & $\begin{array}{l}\text { Warm-needling } \\
\text { moxibustion }\end{array}$ & Ibuprofen & $4 / 4$ & Total effective rate & NR \\
\hline $\begin{array}{l}\text { Kim et al. } \\
{[38]}\end{array}$ & $\begin{array}{l}212(102 / \\
110)\end{array}$ & ACR & Moxibustion & UC & $5 / 5$ & WOMAC & NR \\
\hline $\begin{array}{l}\text { Zhao et al. } \\
\text { [39] }\end{array}$ & $\begin{array}{c}110(55 / \\
55)\end{array}$ & ACR & Moxibustion & $\begin{array}{c}\text { Placebo } \\
\text { moxibustion }\end{array}$ & $6 / 6$ & WOMAC & NR \\
\hline Zhang [40] & $80(40 / 40)$ & ACR & $\begin{array}{l}\text { Warm-needling } \\
\text { moxibustion }\end{array}$ & $\begin{array}{l}\text { Diclofenac } \\
\text { sodium }\end{array}$ & $3 / 3$ & Total effective rate, WOMAC & NR \\
\hline Xu et al. [41] & $60(30 / 30)$ & ACR & $\begin{array}{l}\text { Warm-needling } \\
\text { moxibustion }\end{array}$ & $\begin{array}{l}\text { Diclofenac } \\
\text { sodium }\end{array}$ & $3 / 3$ & Total effective rate, HSS & NR \\
\hline $\begin{array}{l}\text { Jiang et al. } \\
{[42]}\end{array}$ & $90(42 / 44)$ & ACR & $\begin{array}{l}\text { Warm-needling } \\
\text { moxibustion }\end{array}$ & $\begin{array}{l}\text { Glucosamine } \\
\text { sulfate tablets }\end{array}$ & $8 / 8$ & $\begin{array}{c}\text { Total effective rate, WOMAC, } \\
\text { knee flexion }\end{array}$ & $\begin{array}{l}\mathrm{T}: 3 \\
\mathrm{C}: 1\end{array}$ \\
\hline Song et al. & $80(40 / 40)$ & 2 & Sanqi cake moxibustion & $\begin{array}{l}\text { Diclofenac } \\
\text { sodium }\end{array}$ & $3 / 3$ & Total effective rate, WOMAC & NR \\
\hline Qiu [44] & $74(36 / 38)$ & ACR & $\begin{array}{l}\text { Warm-needling } \\
\text { moxibustion }\end{array}$ & Ibuprofen & $4 / 4$ & Total effective rate & NR \\
\hline $\begin{array}{l}\text { Ren and Le } \\
{[45]}\end{array}$ & $\begin{array}{l}150(50 / \\
50 / 50)\end{array}$ & 1 & $\begin{array}{l}\text { Warm-needling } \\
\text { moxibustion }\end{array}$ & $\begin{array}{c}\text { Sodium } \\
\text { hyaluronate }\end{array}$ & $3 / 3$ & $\begin{array}{l}\text { Total effective rate, VAS, } \\
\text { Lysholm, Symptom score }\end{array}$ & NR \\
\hline $\begin{array}{l}\text { Hong et al. } \\
{[46]}\end{array}$ & $78(38 / 37)$ & ACR & Crude herb moxibustion & $\begin{array}{l}\text { Glucosamine } \\
\text { sulfate }\end{array}$ & $12 / 6$ & $\begin{array}{c}\text { Total effective rate, Symptom } \\
\text { score }\end{array}$ & $\begin{array}{l}\mathrm{T}: 2, \\
\mathrm{C}: 1\end{array}$ \\
\hline $\operatorname{Lin}[47]$ & $64(31 / 31)$ & ACR & Crude herb moxibustion & $\begin{array}{l}\text { Glucosamine } \\
\text { sulfate }\end{array}$ & $12 / 6$ & $\begin{array}{c}\text { Total effective rate, WOMAC, } \\
\text { Symptom score }\end{array}$ & $\begin{array}{l}\mathrm{T}: 1 \\
\mathrm{C}: 1\end{array}$ \\
\hline
\end{tabular}


TABLE 2: Continued.

\begin{tabular}{|c|c|c|c|c|c|c|c|}
\hline \multirow{2}{*}{ Study ID } & \multirow{2}{*}{$\begin{array}{c}\text { Sample } \\
\text { size T/C }\end{array}$} & \multirow{2}{*}{$\begin{array}{c}\text { Diagnostic } \\
\text { criteria }\end{array}$} & \multicolumn{2}{|c|}{ Intervention } & \multirow{2}{*}{$\begin{array}{l}\text { Period } \\
(\mathrm{w})\end{array}$} & \multirow{2}{*}{ Outcome } & \multirow{2}{*}{$\begin{array}{c}\text { Drop } \\
\text { out }\end{array}$} \\
\hline & & & Trial group & Control group & & & \\
\hline $\begin{array}{l}\text { Yang et al. } \\
{[48]}\end{array}$ & $\begin{array}{c}120 \\
(60 / 60)\end{array}$ & ACR & $\begin{array}{l}\text { Warm-needling } \\
\text { moxibustion }\end{array}$ & Celecoxib & $4 / 4$ & $\begin{array}{c}\text { Total effective rate, VAS, } \\
\text { Lequesne }\end{array}$ & NR \\
\hline $\begin{array}{l}\text { Ren et al. } \\
{[49]}\end{array}$ & $65(31 / 28)$ & ACR & Moxibustion & $\begin{array}{c}\text { Placebo } \\
\text { moxibustion }\end{array}$ & $6 / 6$ & WOMAC & $\begin{array}{l}\mathrm{T}: 2 \\
\mathrm{C}: 4\end{array}$ \\
\hline $\begin{array}{l}\text { Wu and } \\
\text { Xiong [50] }\end{array}$ & $50(24 / 26)$ & 1 & Heat-sensitive moxibustion & $\begin{array}{c}\text { Sodium } \\
\text { hyaluronate }\end{array}$ & $3 / 3$ & $\begin{array}{c}\text { Total effective rate, Symptom } \\
\text { score }\end{array}$ & NR \\
\hline Fu et al. [51] & $68(37 / 31)$ & ACR & Moxibustion & $\begin{array}{c}\text { Sodium } \\
\text { hyaluronate }\end{array}$ & $24 / 24$ & $\begin{array}{c}\text { Total effective rate, VAS, } \\
\text { Lysholm }\end{array}$ & NR \\
\hline $\begin{array}{l}\text { Ren et al. } \\
{[52]}\end{array}$ & $\begin{array}{c}100 \\
(50 / 50)\end{array}$ & 1 & HCPM & $\begin{array}{l}\text { Diclofenac } \\
\text { sodium }\end{array}$ & $3 / 3$ & Total effective rate & NR \\
\hline $\begin{array}{l}\text { Ding et al. } \\
\text { [53] }\end{array}$ & $\begin{array}{l}90(30 / \\
30 / 30)\end{array}$ & ACR & $\begin{array}{l}\text { Warm-needling } \\
\text { moxibustion }\end{array}$ & Ibuprofen & $2 / 2$ & Total effective rate, WOMAC & NR \\
\hline $\begin{array}{l}\text { Nie et al. } \\
{[54]}\end{array}$ & $\begin{array}{c}116 \\
(58 / 58)\end{array}$ & 1 & Thunder fire moxibustion & Ibuprofen & $5 / 5$ & Total effective rate & NR \\
\hline Zhang [55] & $60(30 / 30)$ & 2 & Moxibustion & $\begin{array}{l}\text { Diclofenac } \\
\text { sodium }\end{array}$ & $2 / 2$ & Total effective rate, Lequesne & NR \\
\hline $\begin{array}{l}\text { Sun et al. } \\
{[56]}\end{array}$ & $60(29 / 27)$ & 1 & SACM & $\begin{array}{l}\text { Diclofenac } \\
\text { sodium }\end{array}$ & $3 / 3$ & $\begin{array}{c}\text { Total effective rate, Symptom } \\
\text { score }\end{array}$ & $\begin{array}{l}\mathrm{T}: 1 \\
\mathrm{C}: 3\end{array}$ \\
\hline $\begin{array}{l}\text { Wu et al. } \\
\text { [57] }\end{array}$ & $\begin{array}{c}114 \\
(60 / 54)\end{array}$ & ACR & Moxibustion & $\begin{array}{l}\text { Diclofenac } \\
\text { sodium }\end{array}$ & $3 / 3$ & Total effective rate & NR \\
\hline
\end{tabular}

Note: 1 = guiding principles of clinical research on new drugs of traditional Chinese medicine; 2 = guidelines for diagnosis and treatment of osteoarthritis 2007/2010; ACR = American College of Rheumatology; UC = regimen performed according to own intention; NR= not reported; AKS = American Knee Society Knee score; VAS = Visual Analogue scale; LKSS = Lysholm knee score; HSS = hospital for special surgery knee score; SF-36 scale = short form 36 questionnaire; WOMAC = Western Ontario and McMaster Universities Osteoarthritis Index; HCPM=herb cake-partitioned moxibustion; SACM $=$ separated aconite cake mild moxibustion.

significant effect of moxibustion on $\mathrm{KOA}(\mathrm{MD}=-0.81,95 \%$ $\mathrm{CI}=-1.24$ to $\left.-0.37, I^{2}=50 \%, P=0.06\right)$.

3.4.5. Lysholm Score. The forest plot illustrating the results of the meta-analysis for the Lysholm score is shown in Figure 8. Five RCTs compared the effectiveness of moxibustion versus western medicine alone and showed a significant effect of moxibustion on $\mathrm{KOA}(\mathrm{MD}=7.61,95 \%$ $\mathrm{CI}=6.04$ to $\left.9.17, I^{2}=95 \%, P<0.00001\right)$.

3.4.6. Lequesne Score. The forest plot illustrating the results of the meta-analysis for the Lequesne score is shown in Figure 9. Three RCTs compared the effects of moxibustion versus western medicine alone and showed a significant effect of moxibustion on $\mathrm{KOA}(\mathrm{MD}=3.29,95 \% \mathrm{CI}=2.93$ to $\left.3.65, I^{2}=99 \%, P<0.00001\right)$.

3.4.7. Adverse Reactions. Adverse reactions were reported in 13 trials included in the study. Four trials [25, 35, 42, 44] reported that no significant adverse reactions or accidents occurred in each group. Wang et al. [26] reported that 1 case of slight scald occurred in the treatment group. Ren et al. [30] reported that there were 22 patients suffered from blisters of different sizes from the moxibustion. Sit and Zhao $[31,39]$ reported that ten patients developed skin flushing at the treated sites after real moxibustion. Without any medical measures, the flush disappears naturally within three days. Kim et al. [38] reported that there were 121 AEs in the treatment group. Hong et al. [46] reported that there were 2 cases of blisters in the treatment group and 6 cases of gastrointestinal discomfort in the control group. Qin [47] reported that there were 2 cases of blisters in the treatment group. Yang et al. [48] reported that there were 9 cases of adverse reactions in the control group and 2 cases of minor scald blisters and 2 cases of local pain at the acupuncture site in the treatment group. Ding et al. [53] reported that epigastric discomfort was found in 3 patients in the western medicine group, and there were no adverse events in the treatment group. Therefore, the adverse reactions in the moxibustion group were considered acceptable by the patients, and it did not affect the statistics of the final result data. Only three trials $[46,48,53]$ documented the specific number of adverse events of two groups. There was no heterogeneity in the 3 trials $\left(P=0.77, I^{2}=0 \%\right)$. The forest plot illustrating the results of the meta-analysis for adverse reactions is shown in Figure 10, and three trials showed no statistical difference between two groups of moxibustion in the treatment of $\mathrm{KOA}(\mathrm{RR}=0.35,95 \% \mathrm{CI}=0.15$ to 0.84 , $\left.I^{2}=0 \%, P=0.77\right)$.

3.5. Study Heterogeneity. $I^{2}$ values were $<50 \%$ for the following outcomes: the total effective rate $\left(I^{2}=45 \%\right)$, WOMAC scale when moxibustion VS negative control $\left(I^{2}=0 \%\right)$ and adverse reactions $\left(I^{2}=0 \%\right) . I^{2}$ values were $\geq 50 \%$ (indicating moderate or substantial heterogeneity) for the following outcomes: WOMAC scale when moxibustion VS western medicine $\left(I^{2}=98 \%\right)$, VAS score $\left(I^{2}=98 \%\right)$, Symptom score $\left(I^{2}=50 \%\right)$, Lysholm score $\left(I^{2}=95 \%\right)$, and Lequesne score $\left(I^{2}=99 \%\right)$. 
TABLE 3: Risk of bias in the included RCTs.

\begin{tabular}{|c|c|c|c|c|c|c|c|}
\hline \multirow[b]{2}{*}{ Study } & \multirow[b]{2}{*}{$\begin{array}{l}\text { Random sequence } \\
\text { generation }\end{array}$} & \multirow[b]{2}{*}{$\begin{array}{c}\text { Allocation } \\
\text { concealment }\end{array}$} & \multicolumn{2}{|c|}{ Blinding } & \multirow[b]{2}{*}{$\begin{array}{l}\text { Outcome data } \\
\text { integrity }\end{array}$} & \multirow[b]{2}{*}{$\begin{array}{l}\text { Selective outcome } \\
\text { reporting }\end{array}$} & \multirow[b]{2}{*}{$\begin{array}{l}\text { Other } \\
\text { biases }\end{array}$} \\
\hline & & & $\begin{array}{l}\text { Patient/doctor } \\
\text { blinding }\end{array}$ & $\begin{array}{c}\text { Outcome assessor } \\
\text { blinding }\end{array}$ & & & \\
\hline Dai et al. [2] & $\begin{array}{c}\text { Random number } \\
\text { table }\end{array}$ & Uncertain & Uncertain & Uncertain & Low risk & Uncertain & Uncertain \\
\hline $\mathrm{Bu}$ et al. [20] & $\begin{array}{l}\text { Random number } \\
\text { table }\end{array}$ & Uncertain & Uncertain & Uncertain & Uncertain & Uncertain & Uncertain \\
\hline Yao [21] & $\begin{array}{l}\text { Random number } \\
\text { table }\end{array}$ & Uncertain & Uncertain & Uncertain & Uncertain & Uncertain & Uncertain \\
\hline Chao [22] & Draw random & Uncertain & Uncertain & Uncertain & Uncertain & Uncertain & Uncertain \\
\hline Li et al. [23] & Random word & High risk & Uncertain & Uncertain & Uncertain & Uncertain & Uncertain \\
\hline Ren [24] & Random word & High risk & Uncertain & Uncertain & Uncertain & Uncertain & Uncertain \\
\hline $\begin{array}{l}\text { Chen et al. } \\
{[25]}\end{array}$ & Random word & High risk & Uncertain & Uncertain & Low risk & Uncertain & Uncertain \\
\hline $\begin{array}{l}\text { Wang et al. } \\
{[26]}\end{array}$ & Computer random & Low risk & Uncertain & Uncertain & Low risk & Uncertain & Uncertain \\
\hline $\begin{array}{l}\text { Huang and Ji } \\
\text { [27] }\end{array}$ & Random word & High risk & Uncertain & Uncertain & Uncertain & Uncertain & Uncertain \\
\hline $\begin{array}{l}\text { Chi et al. } \\
\text { [28] }\end{array}$ & $\begin{array}{c}\text { Random number } \\
\text { table }\end{array}$ & Uncertain & Uncertain & Uncertain & Uncertain & Uncertain & Uncertain \\
\hline Ji [29] & Random word & High risk & Uncertain & Uncertain & Uncertain & Uncertain & Uncertain \\
\hline $\begin{array}{l}\text { Ren et al. } \\
{[30]}\end{array}$ & Computer random & Low risk & Uncertain & Low risk & Low risk & Uncertain & Uncertain \\
\hline Sit et al. [31] & Random word & High risk & Low risk & Uncertain & Uncertain & Uncertain & Uncertain \\
\hline Cui [32] & $\begin{array}{c}\text { Random number } \\
\text { table }\end{array}$ & Uncertain & Uncertain & Uncertain & Low risk & Uncertain & Uncertain \\
\hline $\begin{array}{l}\text { Huang et al. } \\
\text { [33] }\end{array}$ & $\begin{array}{l}\text { Random number } \\
\text { table }\end{array}$ & Uncertain & Uncertain & Uncertain & Uncertain & Uncertain & Uncertain \\
\hline $\begin{array}{l}\text { Zhang and } \\
\mathrm{Li}[34]\end{array}$ & Random word & High risk & Uncertain & Uncertain & Uncertain & Uncertain & Uncertain \\
\hline Wang [35] & Draw random & Uncertain & Uncertain & Uncertain & Uncertain & Uncertain & Uncertain \\
\hline $\begin{array}{l}\text { Deng et al. } \\
{[36]}\end{array}$ & $\begin{array}{l}\text { Random number } \\
\text { table }\end{array}$ & Uncertain & Uncertain & Uncertain & Uncertain & Uncertain & Uncertain \\
\hline Li et al. [37] & Random word & High risk & Uncertain & Uncertain & Low risk & Uncertain & Uncertain \\
\hline $\begin{array}{l}\text { Kim et al. } \\
{[38]}\end{array}$ & Computer random & Low risk & Uncertain & Uncertain & Low risk & Uncertain & Uncertain \\
\hline $\begin{array}{l}\text { Zhao et al. } \\
\text { [39] }\end{array}$ & Computer random & Low risk & Low risk & Low risk & Low risk & Uncertain & Uncertain \\
\hline Zhang [40] & Computer random & Low risk & Single blind & Uncertain & Uncertain & Uncertain & Uncertain \\
\hline $\mathrm{Xu}[41]$ & Random word & High risk & Uncertain & Uncertain & Uncertain & Uncertain & Uncertain \\
\hline $\begin{array}{l}\text { Jiang et al. } \\
\text { [42] }\end{array}$ & $\begin{array}{l}\text { Random number } \\
\text { table }\end{array}$ & Uncertain & Uncertain & Uncertain & Low risk & Uncertain & Uncertain \\
\hline $\begin{array}{l}\text { Song et al. } \\
{[43]}\end{array}$ & Random word & High risk & Uncertain & Uncertain & Uncertain & Uncertain & Uncertain \\
\hline Qiu [44] & $\begin{array}{c}\text { Random number } \\
\text { table }\end{array}$ & Uncertain & Uncertain & Uncertain & Low risk & Uncertain & Uncertain \\
\hline $\begin{array}{l}\text { Ren and } \mathrm{Li} \\
{[45]}\end{array}$ & $\begin{array}{c}\text { Random number } \\
\text { table }\end{array}$ & Uncertain & Uncertain & Uncertain & Uncertain & Uncertain & Uncertain \\
\hline $\begin{array}{l}\text { Hong et al. } \\
{[46]}\end{array}$ & Draw random & Uncertain & Uncertain & Uncertain & Low risk & Uncertain & Uncertain \\
\hline Lin [47] & Draw random & Uncertain & Uncertain & Uncertain & Low risk & Uncertain & Uncertain \\
\hline $\begin{array}{l}\text { Yang et al. } \\
\text { [48] }\end{array}$ & $\begin{array}{c}\text { Random number } \\
\text { table }\end{array}$ & Uncertain & Uncertain & Uncertain & Uncertain & Uncertain & Uncertain \\
\hline $\begin{array}{l}\text { Ren et al. } \\
{[49]}\end{array}$ & Computer random & Low risk & Low risk & Low risk & Low risk & Uncertain & Uncertain \\
\hline $\begin{array}{l}\text { Wu and } \\
\text { Xiong [50] }\end{array}$ & $\begin{array}{l}\text { Random number } \\
\text { table }\end{array}$ & Uncertain & Uncertain & Uncertain & Uncertain & Uncertain & Uncertain \\
\hline Fu et al. [51] & $\begin{array}{c}\text { Random number } \\
\text { table }\end{array}$ & Uncertain & Uncertain & Uncertain & Uncertain & Uncertain & Uncertain \\
\hline $\begin{array}{l}\text { Ren et al. } \\
\text { [52] }\end{array}$ & Random word & High risk & Uncertain & Uncertain & Uncertain & Uncertain & Uncertain \\
\hline
\end{tabular}


TABLE 3: Continued.

\begin{tabular}{|c|c|c|c|c|c|c|c|}
\hline \multirow[b]{2}{*}{ Study } & \multirow[b]{2}{*}{$\begin{array}{c}\text { Random sequence } \\
\text { generation }\end{array}$} & \multirow[b]{2}{*}{$\begin{array}{c}\text { Allocation } \\
\text { concealment }\end{array}$} & \multicolumn{2}{|c|}{ Blinding } & \multirow[b]{2}{*}{$\begin{array}{l}\text { Outcome data } \\
\text { integrity }\end{array}$} & \multirow[b]{2}{*}{$\begin{array}{l}\text { Selective outcome } \\
\text { reporting }\end{array}$} & \multirow[b]{2}{*}{$\begin{array}{l}\text { Other } \\
\text { biases }\end{array}$} \\
\hline & & & $\begin{array}{l}\text { Patient/doctor } \\
\text { blinding }\end{array}$ & $\begin{array}{c}\text { Outcome assessor } \\
\text { blinding }\end{array}$ & & & \\
\hline $\begin{array}{l}\text { Ding et al. } \\
\text { [53] }\end{array}$ & $\begin{array}{l}\text { Random number } \\
\text { table }\end{array}$ & Low risk & Uncertain & Uncertain & Uncertain & Uncertain & Uncertain \\
\hline $\begin{array}{l}\text { Nie et al. } \\
{[54]}\end{array}$ & Random word & High risk & Uncertain & Uncertain & Uncertain & Uncertain & Uncertain \\
\hline Zhang [55] & $\begin{array}{l}\text { Random number } \\
\text { table }\end{array}$ & Uncertain & Uncertain & Uncertain & Uncertain & Uncertain & Uncertain \\
\hline $\begin{array}{l}\text { Sun et al. } \\
{[56]}\end{array}$ & $\begin{array}{c}\text { Random number } \\
\text { table }\end{array}$ & Uncertain & Uncertain & Uncertain & Low risk & Uncertain & Uncertain \\
\hline $\begin{array}{l}\text { Wu et al. } \\
\text { [57] }\end{array}$ & Random word & High risk & Uncertain & Uncertain & Uncertain & Uncertain & Uncertain \\
\hline
\end{tabular}

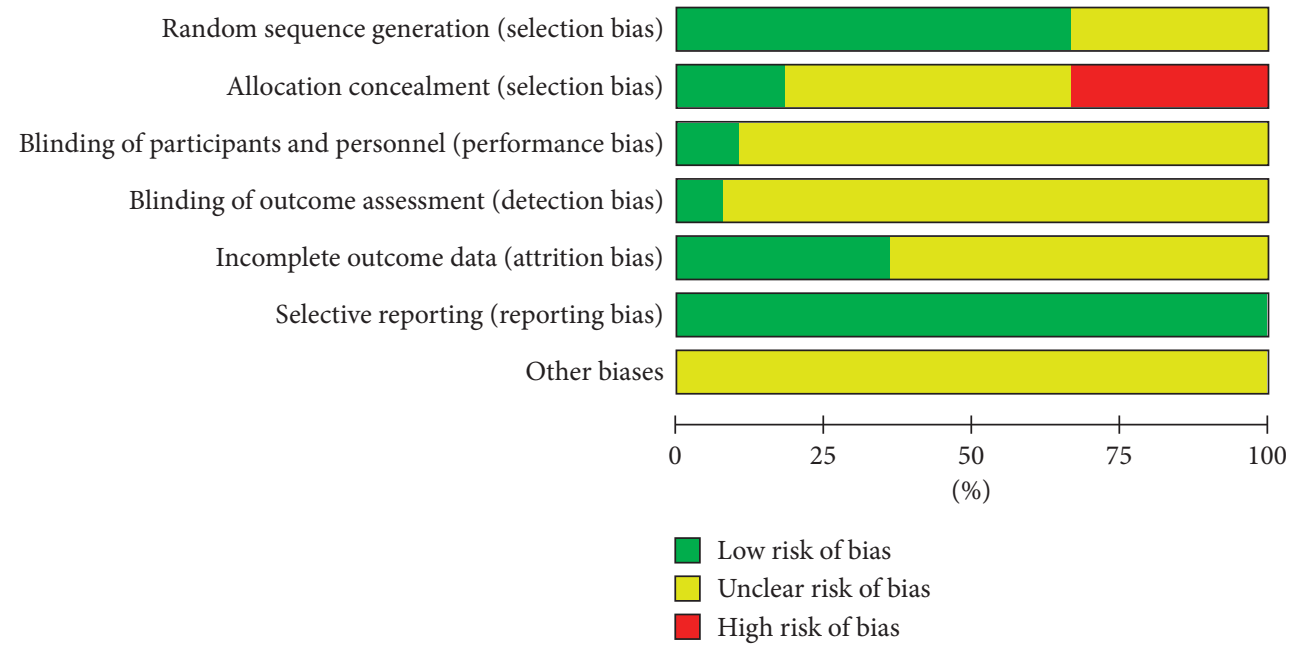

FIgURE 2: Risk of bias graph.

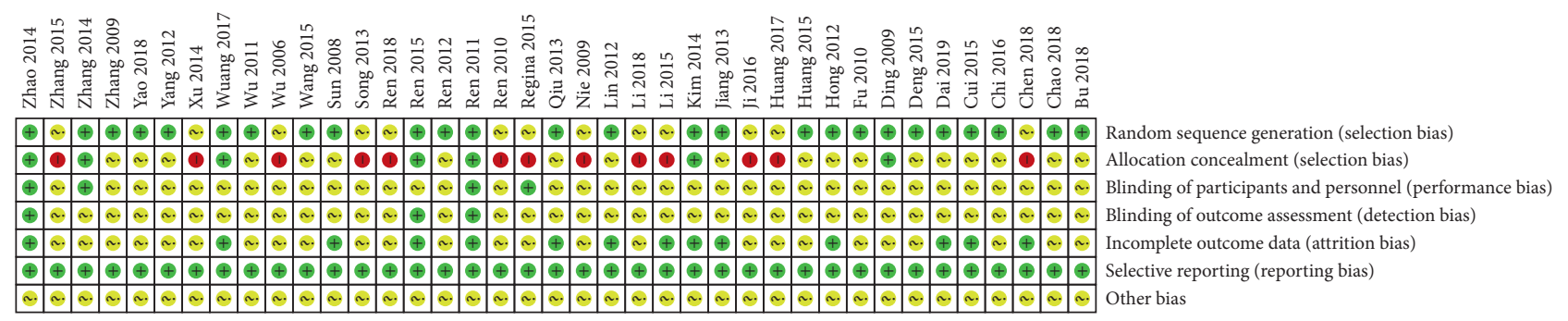

Figure 3: Risk of bias summary.

3.6. Publication Bias. Based on the total effective rate and WOMAC scale of RevMan5.3.0 software, we used a funnel plot to have a qualitative analysis of publication bias. The distribution of graphical cues was not symmetric, and four points (Figure 11) and eight points (Figure 12) were distributed beyond the funnel, indicating that there might be publication bias in our study that influenced the results of our analysis, as shown in Figures 11 and 12.

3.7. Subgroup Analyses. The results of subgroup analysis were summarized for the total effect of moxibustion treatment on KOA. The subgroup analysis showed that there were significant differences within subgroups based on the quality of all included studies and sorts of western medicine and period. $I^{2}$ values were $<50 \%$ and $P$ value $>0.1$ for the following subgroups: quality and period. $I^{2}$ values were $\geq 50 \%$ and $P$ value $<0.1$ for the following subgroup: sorts of western medicine. Therefore, the quality of included studies was regarded as the source of methodological heterogeneity and the period was regarded as the source of clinical heterogeneity. On the contrary, because the heterogeneity of western medicine was still high, we changed the fixed effect model into a randomized effect model. The results showed 


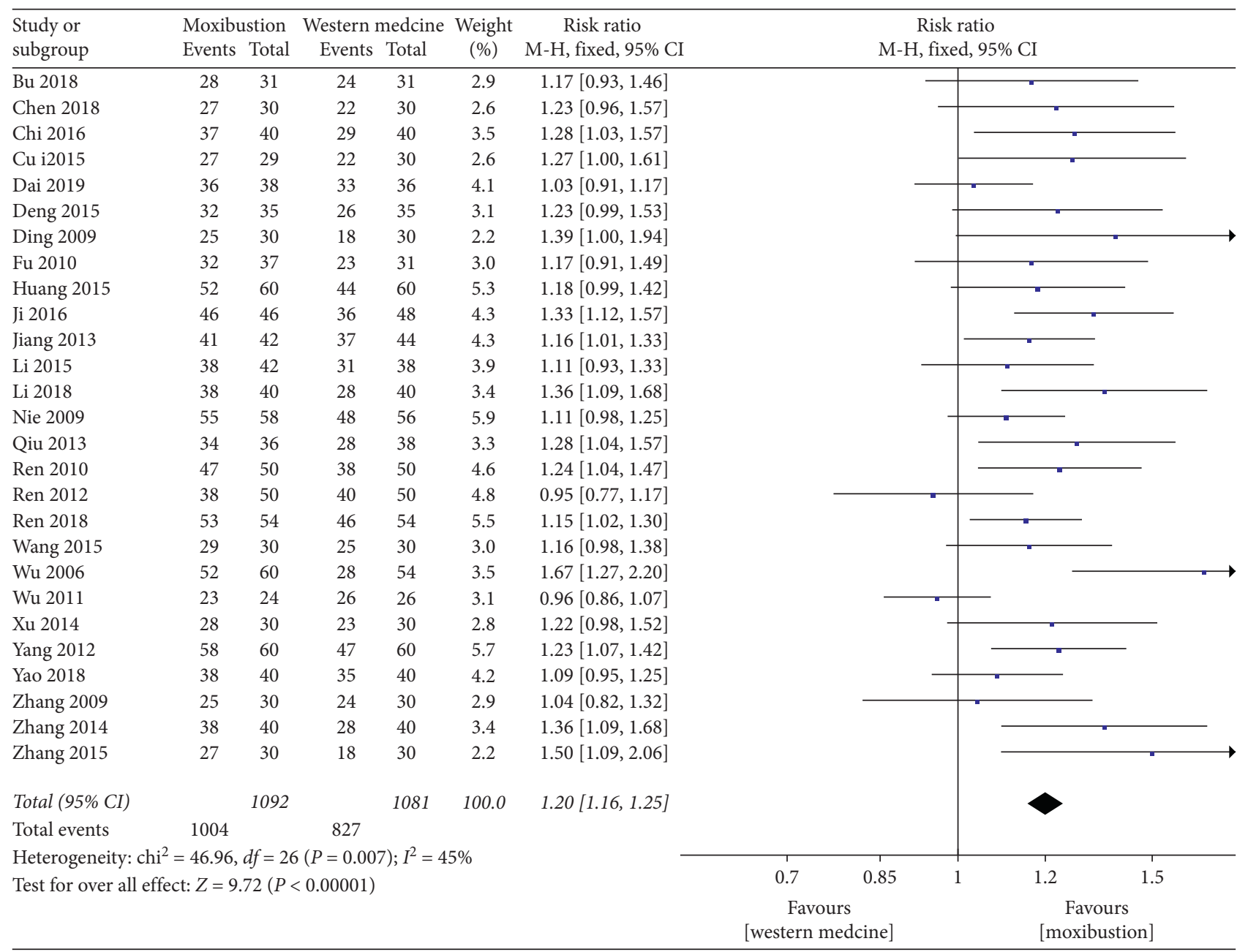

FIgURE 4: Effects of moxibustion according to the total effective rate.

a decrease in heterogeneity $\left(P=0.34, I^{2}=12.0 \%\right)$, which indicated that the sorts of western medicine were regarded as the source of statistical heterogeneity, as shown in Table 4.

3.8. Sensitivity Analyses. We found that the results of heterogeneity comparing the WOMAC scale on moxibustion VS western medicine, VAS score, and Lysholm score were not significantly reduced by omitting the study sequentially. However, the results of heterogeneity comparing the total effective rate significantly reduced $(\mathrm{RR}=1.21,95 \% \mathrm{CI}=1.17$ to $1.26, P=0.16, I^{2}=22 \%$ ) after excluding the $\mathrm{Wu}$ and Xiong [50] study. Therefore, the Wu 2011 [50] study was regarded as the source of heterogeneity. Similarly, the results of heterogeneity comparing symptom score significantly reduced $(\mathrm{MD}=-1.42,95 \% \mathrm{CI}=-1.99$ to $-0.85, P=0.95$, $I^{2}=0 \%$ ) after excluding the Ren and $\mathrm{Li}[45]$ study. Therefore, the Ren and Li [45] study was regarded as the source of heterogeneity. And the results of heterogeneity comparing Lequesne score significantly reduced $(\mathrm{MD}=-0.74,95 \%$ $\mathrm{CI}=-1.43$ to $-0.05, P=0.38, I^{2}=0 \%$ ) after excluding the $\mathrm{Li}$ et al. [23] study. Therefore, the Li et al. [23] study was regarded as the source of heterogeneity, as shown in Table 5.

\section{Discussion}

4.1. Moxibustion Intervention Mechanism. Moxibustion therapy can reduce cartilage damage and macrophage infiltration, improve local blood circulation in the knee joint by inhibiting the expression of inflammatory factors such as mast cell cyclooxygenase, interleukin-6, and tumor necrosis factor, and repair articular chondrocytes [58-61]. Relevant animal experiments showed that moxibustion could increase the limb pedal strength of knee joints of rats by regulating transformed growth factor and insulin-like growth factor [62]. At present, there are few studies on the definite mechanism of moxibustion in patients with KOA. Therefore, it is necessary to conduct more experimental studies on moxibustion intervention in KOA to better guide clinical practice.

4.2. Main Findings of Moxibustion Intervention Effects. The results of this meta-analysis showed a significant total effective rate of moxibustion on $\mathrm{KOA}(\mathrm{RR}=1.20,95 \%$ $\mathrm{CI}=1.16$ to $\left.1.25, I^{2}=45 \%, P=0.007\right)$. In addition, moxibustion intervention also showed significant differences in 


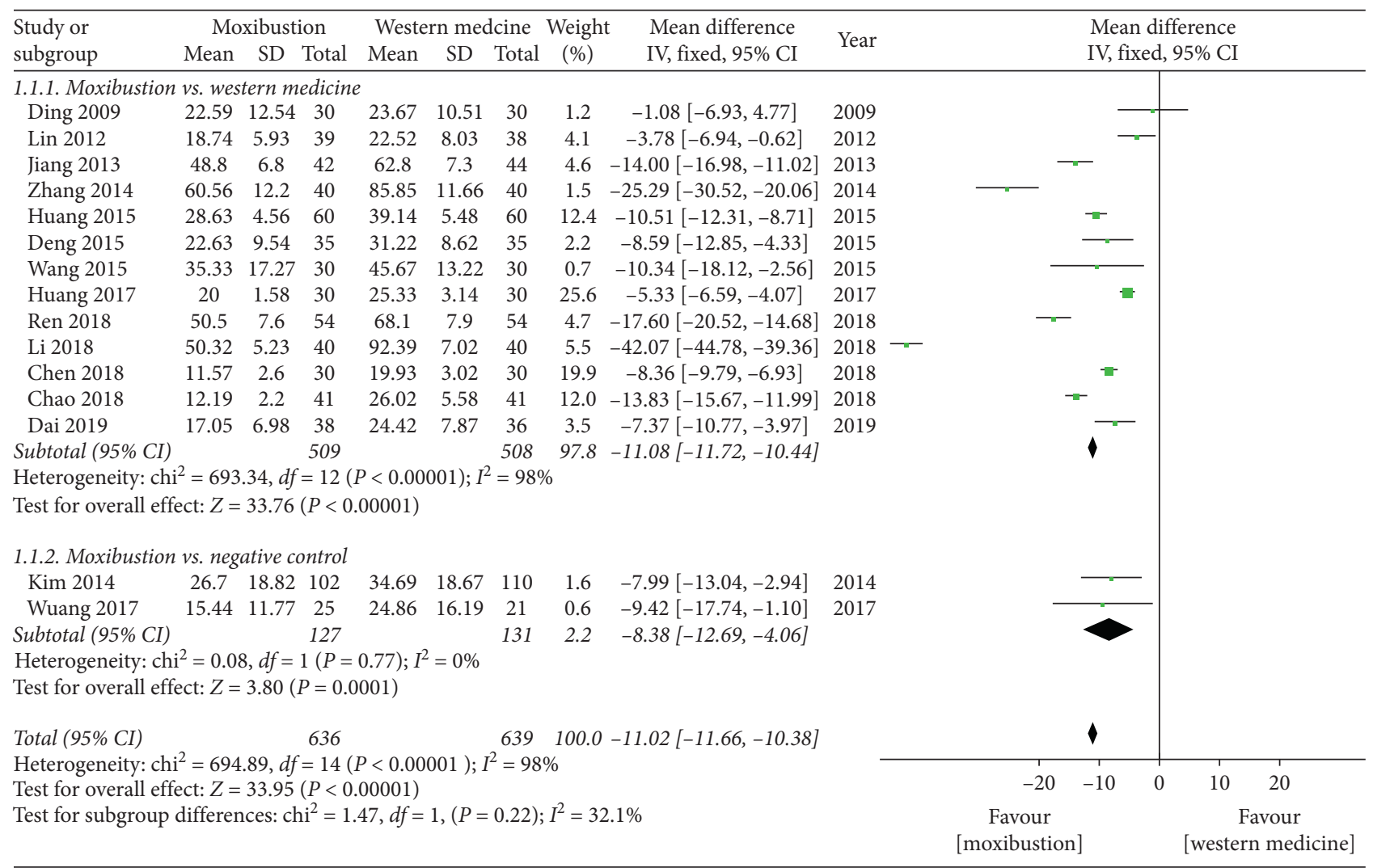

FIGURE 5: Effects of moxibustion according to the WOMAC scale.

\begin{tabular}{|c|c|c|c|c|c|c|c|c|c|c|c|c|c|}
\hline \multirow{2}{*}{$\begin{array}{l}\text { Study or } \\
\text { subgroup }\end{array}$} & \multicolumn{3}{|c|}{ Moxibustion } & \multicolumn{3}{|c|}{ Western medcine } & \multirow{2}{*}{$\begin{array}{c}\text { Weight } \\
(\%)\end{array}$} & \multirow{2}{*}{$\begin{array}{l}\text { Mean difference } \\
\text { IV, fixed, } 95 \% \text { CI }\end{array}$} & \multirow{2}{*}{ Year } & \multirow{2}{*}{\multicolumn{4}{|c|}{$\begin{array}{l}\text { Mean difference } \\
\text { IV, fixed, } 95 \% \text { CI }\end{array}$}} \\
\hline & Mean & SD & Total & Mean & SD & Total & & & & & & & \\
\hline Fu 2010 & 3.48 & 1.7 & 37 & 4.12 & 2.6 & 31 & 3.0 & $-0.64[-1.71,0.43]$ & 2010 & & & & \\
\hline Ren 2012 & 4.87 & 1.02 & 50 & 4.71 & 1.03 & 50 & 21.1 & $0.16[-0.24,0.56]$ & 2012 & & 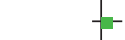 & & \\
\hline Huang 2017 & 1.9 & 1.35 & 30 & 3.07 & 1.41 & 30 & 7.0 & $-1.17[-1.87,-0.47]$ & 2017 & & $\because$ & & \\
\hline Bu 2018 & 2.01 & 0.28 & 31 & 5.76 & 0.71 & 31 & 47.2 & $-3.75[-4.02,-3.48]$ & 2018 & $\square$ & & & \\
\hline Yao 2018 & 3.79 & 1.34 & 40 & 5.28 & 1.46 & 40 & 9.0 & $-1.49[-2.10,-0.88]$ & 2018 & & $\rightarrow$ & & \\
\hline Dai 2019 & 2.48 & 1.03 & 38 & 3.62 & 1.23 & 36 & 12.7 & $-1.14[-1.66,-0.62]$ & 2019 & & - & & \\
\hline Total (95\% CI) & & & 226 & & & 218 & 100.0 & $-2.12[-2.30,-1.93]$ & & & $\diamond$ & & \\
\hline \multicolumn{10}{|c|}{ Heterogeneity: $\mathrm{chi}^{2}=297.37, d f=5(P<0.00001) ; I^{2}=98 \%$} & -4 & -2 & 2 & 4 \\
\hline \multicolumn{10}{|c|}{ Test for overall effect: $Z=22.47(P<0.00001)$} & $\begin{array}{c}-4 \\
\text { Favour } \\
\text { [moxibustion] }\end{array}$ & ] & $\begin{array}{r}2 \\
\text { Fa } \\
\text { [western }\end{array}$ & $\begin{array}{l}4 \\
\text { our } \\
\text { medicine] }\end{array}$ \\
\hline
\end{tabular}

FIgURE 6: Effects of moxibustion according to the VAS score.

WOMAC scores $(\mathrm{MD}=-11.02,95 \% \mathrm{CI}=-11.66$ to -10.38 , $\left.P=0.22, \quad I^{2}=32.1 \%\right), \quad$ VAS score $\quad(\mathrm{MD}=-2.12, \quad 95 \%$ $\mathrm{CI}=-2.30$ to $\left.-1.93, I^{2}=98 \%, P<0.00001\right)$, Symptom score $\left(\mathrm{MD}=-0.81,95 \% \mathrm{CI}=-1.24\right.$ to $\left.-0.37, I^{2}=50 \%, P=0.06\right)$, Lysholm score $\left(\mathrm{MD}=7.61,95 \% \mathrm{CI}=6.04\right.$ to $9.17, I^{2}=95 \%$, $P<0.00001)$, and Lequesne score $(\mathrm{MD}=3.29,95 \% \mathrm{CI}=2.93$ to $\left.3.65, I^{2}=99 \%, P<0.00001\right)$ in patients with KOA. In general, moxibustion intervention can reduce pain and improve knee symptoms of patients with KOA and has fewer adverse reactions. Therefore, moxibustion treatment for KOA is safe and effective, which is worthy of clinical application.
4.3. Quality of Evidence. Among the 39 RCTs were included in this study, containing 3293 patients. The quality of evidence differs from low to high based on the Cochrane Bias Evaluation Tools and Jadad scale. Fourteen trials were of high quality, ten were of moderate quality, and fifteen were of low quality. Therefore, the quality of the included studies was moderate. The inappropriate random method, allocation concealment, and a lack of blinding of most studies exaggerated the results of the outcome measures. In this study, there were $66.67 \%$ of the literature and $17.95 \%$ of the literature correctly reported randomized grouping and allocation of hidden information, respectively. This could lead to overestimate. 


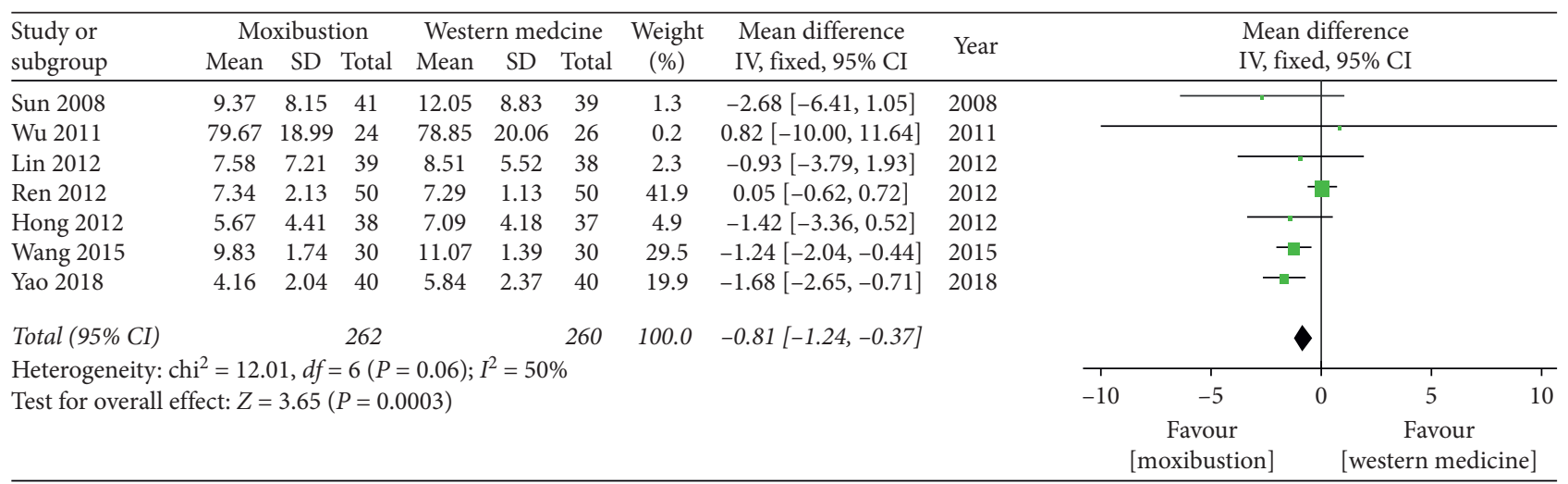

FIgURE 7: Effects of moxibustion according to the Symptom score.

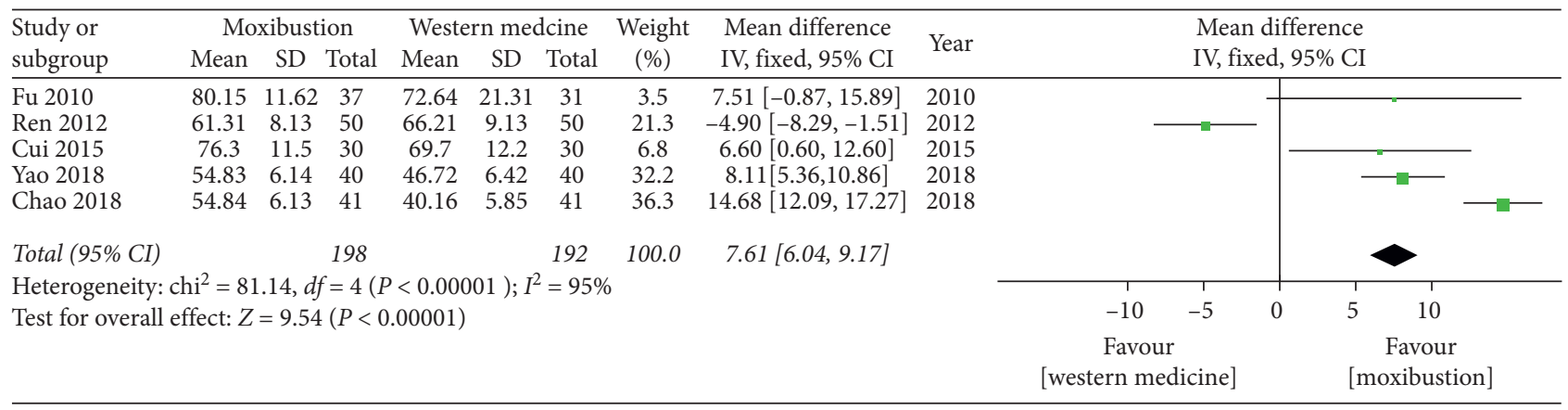

FIGURE 8: Effects of moxibustion according to the Lysholm score.

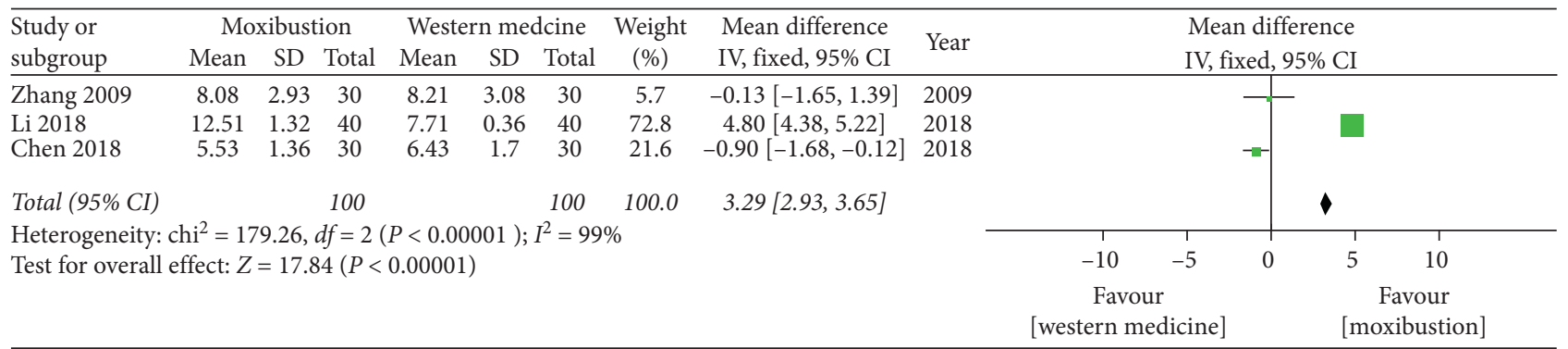

Figure 9: Effects of moxibustion according to the Lequesne score.

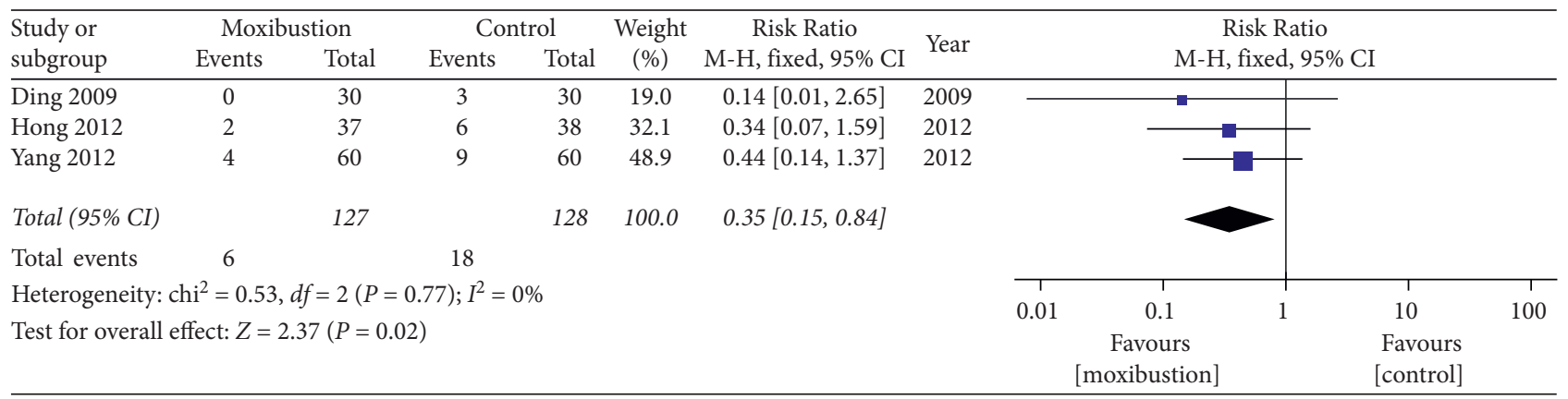

FIgURE 10: Meta-analysis for adverse events of moxibustion.

4.4. Discussion of Heterogeneity. There was heterogeneity in the effectiveness of moxibustion on the total effective rate of KOA. To explore its source, we adopted subgroup analysis and found that clinical heterogeneity was mainly related to the sorts of western medicine, duration of intervention, and other factors, its methodological heterogeneity was mainly 


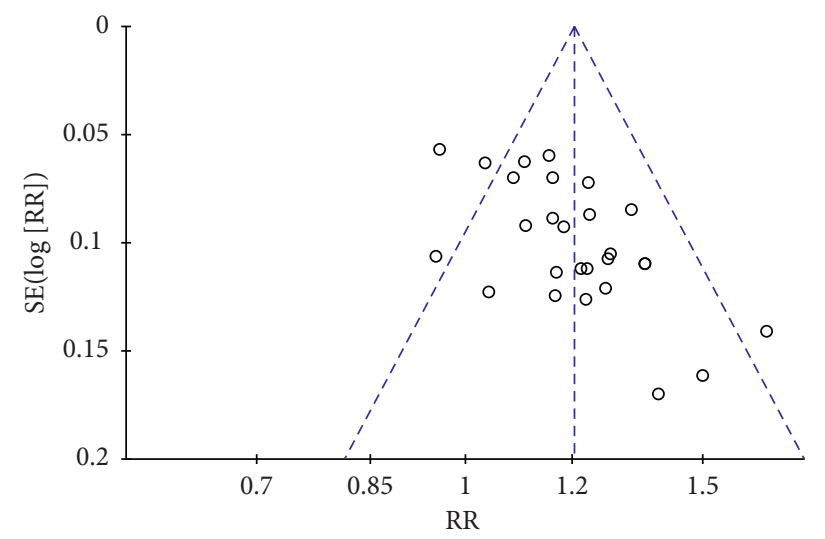

Figure 11: Funnel plot based on total effective rate publication bias.

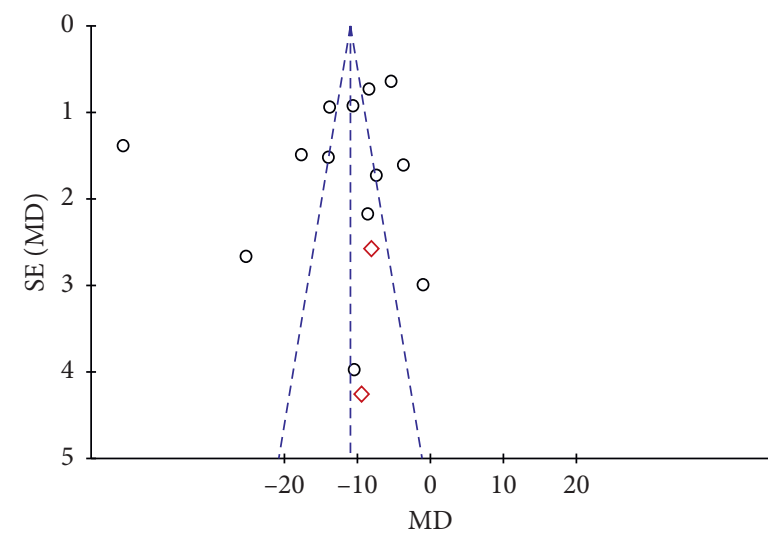

Subgroups

○ Moxibustion vs. western medicine

$\diamond$ Moxibustion vs. negative control

Figure 12: Funnel plot based on WOMAC-scale publication bias.

TABle 4: Subgroup analysis of the total effective rate for each variable.

\begin{tabular}{|c|c|c|c|c|c|c|}
\hline \multirow{2}{*}{ Variable } & \multirow{2}{*}{ No. of trials } & \multicolumn{2}{|c|}{ No. of participants } & \multirow{2}{*}{ RR (95\% CI) } & \multirow{2}{*}{$P$ value } & \multirow{2}{*}{$I^{2}$ value } \\
\hline & & Events & Total & & & \\
\hline \multicolumn{7}{|l|}{ Quality } \\
\hline High quality & 6 & 367 & 433 & $1.23[1.13,1.33]$ & \multirow{2}{*}{0.60} & \multirow{2}{*}{$0 \%$} \\
\hline Low quality & 21 & 1464 & 1740 & $1.20[1.15,1.25]$ & & \\
\hline \multicolumn{7}{|l|}{ Western medicine } \\
\hline Celecoxib & 2 & 174 & 194 & $1.15[1.04,1.27]$ & \multirow{5}{*}{0.08} & \multirow{5}{*}{$52.4 \%$} \\
\hline Glucosamine sulfate & 3 & 210 & 241 & $1.22[1.10,1.34]$ & & \\
\hline Sodium hyaluronate & 5 & 302 & 358 & $1.09[1.00,1.19]$ & & \\
\hline Ibuprofen & 7 & 474 & 558 & $1.20[1.11,1.29]$ & & \\
\hline Diclofenac sodium & 8 & 521 & 654 & $1.29[1.19,1.40]$ & & \\
\hline \multicolumn{7}{|l|}{ Period } \\
\hline Two weeks & 4 & 203 & 260 & $1.31[1.14,1.49]$ & \multirow{4}{*}{0.31} & \multirow{4}{*}{$16.9 \%$} \\
\hline Three weeks & 7 & 458 & 563 & $1.23[1.13,1.33]$ & & \\
\hline Four weeks & 9 & 626 & 740 & $1.19[1.12,1.27]$ & & \\
\hline Eight weeks & 3 & 205 & 226 & $1.13[1.04,1.23]$ & & \\
\hline
\end{tabular}

$P$ value and $I^{2}$ value test for heterogeneity between subgroups.

affected by the quality of the included literature and the subjective bias of literature quality evaluation, and the sorts of western medicine was regarded as the source of statistical heterogeneity. Similarly, by changing the research effect model and adopting the sensitivity analysis method, we found that the heterogeneity was significantly reduced after 
TABLE 5: Summary of sensitivity analysis for the total effective rate, Symptom score, and Lequesne score.

\begin{tabular}{|c|c|c|c|}
\hline $\begin{array}{l}\text { Total } \\
\text { rate }\end{array}$ & $\begin{array}{l}\text { RR fluctuation } \\
\text { (1.20 to } 1.21)\end{array}$ & $\begin{array}{l}P \text { fluctuation } \\
(0.007 \text { to } 0.16)\end{array}$ & $\begin{array}{c}I^{2} \\
\text { fluctuation } \\
(45 \% \text { to } \\
22 \%)\end{array}$ \\
\hline & $\begin{array}{r}\mathrm{N} \\
\text { fluct }\end{array}$ & $P$ fluctuation & $\begin{array}{c}I^{2} \\
\text { fluctuation }\end{array}$ \\
\hline Symp & $\begin{array}{l}(-0.81 \text { to } \\
-1.42)\end{array}$ & (0.06 to 0.95$)$ & $(50 \%$ to $0 \%)$ \\
\hline Lequesne score & $(-0.74$ to 3.29$)$ & $\begin{array}{c}(<0.00001 \text { to } \\
0.38)\end{array}$ & $(99 \%$ to $0 \%)$ \\
\hline
\end{tabular}

omitting the study of $\mathrm{Wu}$ and Xiong [50], Ren and Li [45], and $\mathrm{Li}$ et al. [23]. To explore its reasons, it was found that they all have problems such as low quality, the flaw in test design, or small sample sizes. This indicated that the results of this meta-analysis, to a certain extent, were affected by the risk of bias.

4.5. Limitations and Advantages. There were several limitations of this systematic review and meta-analysis, as follows:

Firstly, the evaluation criteria for the total effective rate were inconsistent. There were eight trials using the WOMAC scale, nine trials using guiding principles of clinical research on new drugs of traditional Chinese medicine, two trials using criteria for diagnosis and effectiveness of TCM diseases, and eleven trials using other criteria to evaluate the clinical efficacy of the patients with KOA. Therefore, it might be one of the sources of heterogeneity. However, due to the limitation of the number of included literature, the lack of integrated data and this review did not distinguish effectiveness criteria. Therefore, it is suggested that clinical trials should be strengthened in future studies, and clinical effectiveness should be evaluated by the internationally unified effectiveness evaluation standards. Thus, the results have more authenticity and reliability.

Secondly, the methodological quality of most included studies was relatively low, and there was a latent risk of bias, to some extent, which weakened the credibility and reliability of the evidence of moxibustion therapy for $\mathrm{KOA}$ in this systematic review and meta-analysis. For example, since few control groups were placebo controls, it was difficult to eliminate the placebo effect. Although the random word appeared in all the included literature, only 26 trials described the correct random method. In most of the included trials, allocation concealment and blind method were not clear, which may lead to potential implementation bias and selectivity bias. Most of the included RCTs were conducted in China, and only seven articles were published in English. And the results of the funnel plot indicated potential publication bias.

Finally, some individual data were incomplete, so we failed to follow the preset subgroup analysis based on variable comparisons. Also, because the necessary data were not available, so patients with either meniscal symptoms or who have degenerative meniscal disease were not discussed as a subgroup within the review. On the contrary, to explore the source of heterogeneity, we performed a subgroup analysis of the total effect based on the quality of all included studies, sorts of western medicine and period.

While this systematic review and meta-analysis had some limitations, it showed some significant advantages. Most importantly, compared with published systematic review and meta-analysis, literature retrieval in this study was more comprehensive with systematic literature retrieval strategy, and the number of included literature was larger. Therefore, the evidence was more reliable and scientific. In addition, subgroup analysis was performed according to the quality of all included studies, sorts of western medicine and period. And we used more adequate outcome indicators to conduct this meta-analysis. Thus, the stability of our research results was demonstrated. Finally, this systematic review and metaanalysis were performed following the PRISMA statement strictly, and the content was more complete.

\section{Conclusion}

In this systematic review and meta-analysis of RCTs, the effectiveness and safety of moxibustion in the treatment of $\mathrm{KOA}$ are positive. But it still needs a well-designed, rigorous, large sample, and multicenter prospective randomized controlled trials on this subject to confirm the validity of the results. In the future, we can also include trials, for example, moxibustion combined with positive controls (e.g., western medicine) VS positive controls (e.g., western medicine) to expand the number of original studies, so as to include higher-quality studies, reduce the influence of publication bias, and improve the credibility of research and better guide clinical practice.

\section{Abbreviations \\ KOA: Knee osteoarthritis \\ CI: Confidence interval \\ MD: Mean difference \\ RR: Risk ratio \\ RCT: Randomized controlled trial \\ UC: Regimen performed according to own intention.}

\section{Additional Points}

S1 PRISMA Checklist. The PRISMA Checklist of this metaanalysis. PRISMA 2009 checklist.doc.

\section{Conflicts of Interest}

The authors declare that they have no conflicts of interest. 


\section{Authors' Contributions}

Jun Xiong, Ting Yuan, Jun Yang, and Xue Wang extracted the data. Ting Yuan prepared the original draft. Jun Xiong reviewed and edited the manuscript.

\section{Acknowledgments}

This work was supported by the National Natural Science Foundation of China (81573835), Jiangxi Youth Science Foundation Key Project (20192ACB21007), and Jiangxi Outstanding Young Talents Funding Scheme, China (Grant number: 20171BCB23093).

\section{References}

[1] S. N. Issa and L. Sharma, "Epidemiology of osteoarthritis: an update," Current Rheumatology Reports, vol. 8, no. 1, pp. 7-15, 2006.

[2] M. Dai, X.-Y. Fang, H.-Y. Chen, Y.-H. Wang, and Y.-W. Wu, "Clinical study on mild moxibustion for knee osteoarthritis," Journal of Acupuncture and Tuina Science, vol. 17, no. 1, pp. 62-66, 2019.

[3] J. Dawson, L. Linsell, K. Zondervan et al., "Impact of persistent hip or knee pain on overall health status in elderly people: a longitudinal population study," Arthritis \& Rheumatism, vol. 53, no. 3, pp. 368-374, 2005.

[4] T. Kawahara, T. Sasho, T. Ohnishi et al., "Stage-specific meniscal features predict progression of osteoarthritis of the knee: a retrospective cohort study using data from the osteoarthritis initiative," BMC Musculoskeletal Disorders, vol. 20, no. 1, 2019.

[5] X. Tang, S. Wang, S. Zhan et al., "The prevalence of symptomatic knee osteoarthritis in China: results from the China health and retirement longitudinal study," Arthritis \& Rheumatology, vol. 68, no. 3, pp. 648-653, 2016.

[6] M. Cross, E. Smith, D. Hoy et al., "The global burden of hip and knee osteoarthritis: estimates from the global burden of disease 2010 study," Annals of the Rheumatic Diseases, vol. 73, no. 7, pp. 1323-1330, 2014.

[7] M. C. Hochberg, R. D. Altman, K. T. April et al., "American College of Rheumatology 2012 recommendations for the use of nonpharmacologic and pharmacologic therapies in osteoarthritis of the hand, hip, and knee," Arthritis Care \& Research, vol. 64, no. 4, pp. 465-474, 2012.

[8] T. E. Towheed, M. J. Judd, M. C. Hochberg et al., "Acetaminophen for osteoarthritis," Cochrane Database of Systematic Reviews, vol. 1, no. 1, Article ID CD004257, 2006.

[9] World Health Organization and Regional Office for the Western Pacific, WHO International Standard Terminologies on Traditional Medicine in the Western Pacific Region, WHO, Geneva, Switzerland, 2007.

[10] L. Luo, M. Liao, J.-X. Peng et al., "Comparison of the efficacy between conventional moxibustion and smoke-free moxibustion on knee osteoarthritis: a randomized controlled trial," Evidence-based Complementary and Alternative Medicine, vol. 2019, Article ID 1291947, 8 pages, 2019.

[11] X. Lu, F. Wang, Z. Yan et al., "Systematic review of clinical effect of moxibustion therapy on patients with knee osteoarthritis," Guangming Chinese Medicine, vol. 34, no. 10, pp. 1615-1620, 2019.

[12] J. Yu and J. Xiong, "Systematic review and meta-analysis of the clinical efficacy of thermal moxibustion in the treatment of knee osteoarthritis," Guangzhou University of Traditional Chinese Medicine, vol. 32, no. 1, pp. 60-66, 2015.

[13] Y. Liu, J. Xu, and M. Lin, "Systematic review and metaanalysis of moxibustion therapy for knee osteoarthritis," Journal of Clinical and Experimental Medicine, vol. 16, no. 8, pp. 783-787, 2017.

[14] Y. Wang, Y. Wang, S. Jing et al., "Meta-analysis of the clinical effect of moxibustion on knee osteoarthritis," Hunan Journal of TCM, vol. 33, no. 9, pp. 148-151, 2017.

[15] A. Li, Z. J. Wei, Y. Liu et al., "Moxibustion treatment for knee osteoarthritis: a systematic review and meta-Analysis," Medicine, vol. 95, no. 14, p. 3244, 2016.

[16] G. M. Song, X. Tian, Y. H. Jin et al., "Moxibustion is an alternative in treating knee osteoarthritis: the evidence from systematic review and meta-analysis," Medicine, vol. 95, no. 6, p. 2790, 2016.

[17] D. Moher, A. Liberati, J. Tetzlaff et al., "Preferred reporting items for systematic reviews and meta-analyses: the PRISMA statement," BMJ, vol. 339, p. b2535, 2009.

[18] A. R. Jadad, R. Andrew Moore, D. Carroll et al., "Assessing the quality of reports of randomized clinical trials: is blinding necessary?," Controlled Clinical Trials, vol. 17, no. 1, 1996.

[19] P. Higgins Julian, A. Altman, J. Sterne et al., "Assessing risk of bias in included studies," in Cochrane Handbook for Systematic Reviews of Interventions: Cochrane Book Series, John Wiley \& Sons, Ltd, Hoboken, NJ, USA, 2011.

[20] A. Bu, L. Wang, J. Xiong et al., "Moxibustion with three volts of heat-sensitive wheat grains for treatment of cold dampness and stasis type osteoarthritis of knee joint," Lishizhen Medicine and Materia Medica Research, vol. 29, no. 5, pp. 11141116, 2018.

[21] X. Yao, "Randomized controlled study of Warm-needling moxibustion for knee osteoarthritis," Inner Mongol Journal of Traditional Chinese Medicine, vol. 37, no. 6, pp. 83-84, 2018.

[22] J. Chao, "A randomized controlled clinical study of Warmneedling moxibustion in the treatment of knee osteoarthritis," Betes World, vol. 15, no. 9, p. 40, 2018.

[23] H.-X. Li, S. Pei, L. Xia et al., "Clinical effect of Warm-needling moxibustion on knee osteoarthritis," Clinical Research and Practice, vol. 3, no. 36, pp. 130-131, 2018.

[24] Z. Ren, "Clinical study on Warm-needling moxibustion treatment of knee osteoarthritis," China Health Standard Management, vol. 9, no. 8, pp. 94-96, 2018.

[25] J. Chen, J. Wang, K. Zhao et al., "Clinical effect observation of Warm-needling moxibustion in treating yang-deficiency and cold-setting knee osteoarthritis," Journal of Shanxi University of Chinese Medicine, vol. 41, no. 6, pp. 88-90, 2018.

[26] X. Wang, X. Wang, M. Hou et al., "Warm-needling moxibustion for knee osteoarthritis: a randomized controlled trial," Zhongguo Zhen Jiu, vol. 37, no. 5, pp. 457-462, 2017.

[27] L. Huang and R. Ji, "Clinical study on the effect of thunder fire moxibustion on VAS and WOMAC scores of patients with knee osteoarthritis," Jiangsu Journal of Traditional Chinese Medicine, vol. 49, no. 8, pp. 57-58, 2017.

[28] Z. Chi, J. Xiong, J. Lin et al., "Moxibustion and thermosensitive law in the treatment of knee osteoarthritis: an exploratory RCT study," Chinese Medicine Modern Distance Education of China, vol. 14, no. 4, pp. 104-106, 2017.

[29] W. Ji, "Clinical observation of 96 cases of knee osteoarthritis treated with warm-needling moxibustion," Medical Frontier, vol. 6, no. 10, pp. 187-188, 2016.

[30] X. Ren, C. Yao, F. Wu et al., "Effectiveness of moxibustion treatment in quality of life in patients with knee osteoarthritis: a randomized, double-blinded, placebo-controlled trial," 
Evidence-based Complementary and Alternative Medicine, vol. 2015, Article ID 569523, 6 pages, 2015.

[31] R. W. S. Sit, X. Y. Wu, S. S. Hung, and V. C. H. Chung, "Is moxibustion useful for improving pain and function amongst patients with osteoarthritis of the knee as compared to sham moxibustion?," Advances in Integrative Medicine, vol. 2, no. 2, pp. 110-111, 2015.

[32] J. Cui, A Randomized Controlled Study on the Intervention of Knee Osteoarthritis by Moxibustion under Warm Acupuncture Box, Nanjing University of Traditional Chinese Medicine, Nanjing, China, 2015.

[33] Z. Huang, S.-L. Song, and G. Huang, "Observation on clinical effects of herbal cake-partitioned moxibustion for knee osteoarthritis," Journal of Acupuncture and Tuina Science, vol. 13, no. 4, pp. 242-245, 2015.

[34] Y. Zhang and X. Li, "Clinical observation on the treatment of 30 cases of knee osteoarthritis with mild moxibustion with "double solid" septum," Zhejiang Journal of Traditional Chinese Medicine, vol. 50, no. 5, p. 382, 2015.

[35] W. Wang, Clinical Research on Moxibustion Treatment of Yang-Deficiency and Cold-Setting Knee Osteoarthritis, Yunnan University of Traditional Chinese Medicine, Kunming, China, 2015.

[36] J. Deng, Y. Chen, and S. Wang, "Thirty-five cases of treatment of knee osteoarthritis with salt moxibustion," Journal of Clinical Acupuncture and Moxibustion, vol. 31, no. 3, pp. 14-17, 2015.

[37] Z. Li, W. Wu, M. Liu et al., "Clinical observation of acupuncture treatment for knee osteoarthritis," World Latest Medicine, vol. 12, pp. 109-110, 2015.

[38] T. H. Kim, K. H. Kim, J. W. Kang et al., "Moxibustion treatment for knee osteoarthritis: a multi-centre, non-blinded, randomized controlled trial on the effectiveness and safety of the moxibustion treatment versus usual care in knee osteoarthritis patients," PLoS One, vol. 9, no. 7, Article ID e101973, 2014.

[39] L. Zhao, K. Cheng, L. Wang et al., "Effectiveness of moxibustion treatment as adjunctive therapy in osteoarthritis of the knee: a randomized, double-blinded, placebo-controlled clinical trial," Arthritis Research and Therapy, vol. 16, no. 3, 2014.

[40] Y. Zhang, Clinical Effect and Related Cytokines of Wen Acupuncture and Moxibustion in Treating Knee Osteoarthritis, Ningxia Medical University, Yinchuan, China, 2014.

[41] $\mathrm{H}$. Xu, "Application of wen acupuncture and moxibustion in the treatment of senile osteoarthritis of the knee joint," Healthy People, vol. 8, no. 4, p. 41, 2014.

[42] B. Jiang, X. Hou, and Z. Tang, "Clinical study of acupuncture and moxibustion in the treatment of knee osteoarthritis," Chinese Journal of Traditional Medical Traumatology \& Orthopedics, vol. 21, no. 11, pp. 18-21, 2013.

[43] Y. Song, D. Liu, and J. Zhu, "Clinical study of moxibustion with notoginseng cake for treatment of knee osteoarthritis with blood stasis," Journal of Clinical Acupuncture and Moxibustion, vol. 29, no. 9, pp. 40-42, 2013.

[44] S. Qiu, "Warm-needling moxibustion of knee osteoarthritis randomized parallel control study," Journal of Practical Traditional Chinese Internal Medicine, vol. 27, no. 14, pp. 86-87, 2013.

[45] J. Ren and T. Li, "Warm-needling moxibustion combined with sodium hyaluronate joint cavity injection for the treatment of knee osteoarthritis," Journal of Xinjiang Medical University, vol. 35, no. 9, pp. 1212-1215, 2012.

[46] K. Hong, L. Li, Q. Que et al., "Clinical study of moxibustion in treating yang-deficiency and cold-setting knee osteoarthritis,"
China Journal of Traditional Chinese Medicine and Pharmacy, vol. 27, no. 8, pp. 2227-2230, 2012.

[47] L. Qin, Clinical Effect Observation of Moxibustion on WindCold Dampness Resistance Type Knee Osteoarthritis, Fujian University of Traditional Chinese Medicine, Fuzhou, China, 2012.

[48] X. Yang, S. He, R. Wang et al., "Effect observation of warm acupuncture muscle stimulation therapy in the treatment of knee osteoarthritis," Acupuncture Research, vol. 37, no. 3, pp. 237-241, 2012.

[49] X. Ren, J. Cao, X. Shen et al., "Treatment of knee osteoarthritis with moxibustion: a randomized controlled study," Zhongguo Zhen Jiu, vol. 31, no. 12, pp. 1057-1061, 2011.

[50] F. Wu and P. Xiong, "Clinical study on the treatment of knee joint osteoarthritis (swelling type) by heat-sensitized acupoints with moxibustion," Journal of Clinical Acupuncture and Moxibustion, vol. 27, no. 11, pp. 1-4, 2011.

[51] X.-M. Fu, Y. Huang, G. Lu et al., "Effect of moxibustion and sodium hyaluronate on the treatment of knee osteoarthritis," Journal of Chinese Physician, vol. 12, no. 3, pp. 425-426, 2010.

[52] X.-M. Ren, S.-L. Song, and Y. Shi, "50 cases of knee osteoarthritis treated with traditional Chinese medicine cake and moxibustion," Zhejiang Journal of Traditional Chinese Medicine, vol. 45, no. 10, p. 759, 2010.

[53] M. Ding, H. Zhang, and Y. Li, "Warm-needling moxibustion in the treatment of osteoarthritis of knee joint: a randomized controlled study," Zhongguo Zhen Jiu, vol. 29, no. 8, pp. 603-607, 2009.

[54] B. Nie, L. Zhang, K. Xu et al., "Clinical observation of Zhao's thunder fire moxibustion in the treatment of knee osteoarthritis," Jilin TCM Journal, vol. 29, no. 4, pp. 313-314, 2009.

[55] S. Zhang, Mild Moxibustion for Treatment of Yangxu Cold Coagulation Type Knee Osteoarthritis, Guangzhou University of Traditional Chinese Medicine, Guangzhou, China, 2009.

[56] K. Sun, J. Yang, and D. K. Shen, "Clinical observation on treatment of primary knee osteoarthritis of liver and kidney deficiency type with Aconite cake-separated moxibustion," Zhongguo Zhen Jiu, vol. 28, no. 2, pp. 87-90, 2008.

[57] Y. Wu, Y. Zhang, and R. Liu, "Warm-needling moxibustion treatment of knee osteoarthritis clinical observation on 60 cases," Journal of New Chinese Medicine, vol. 38, no. 1, pp. 67-66, 2006.

[58] J.-S. Han, "Acupuncture: neuropeptide release produced by electrical stimulation of different frequencies," Trends in Neurosciences, vol. 26, no. 1, pp. 17-22, 2003.

[59] L. Hu, L. Wang, J. Wei et al., "Heat induces adenosine triphosphate release from mast cells in vitro: a putative mechanism for moxibustion," Journal of Traditional Chinese Medicine, vol. 35, no. 3, pp. 323-328, 2015.

[60] X. Li, Z. Li, L. Wang et al., "Effect of mild indirect moxibustion on hemorheology of knee osteoarthritis," Chinese Archives of Traditional Chinese Medicine, vol. 30, no. 9, pp. 2098-2099, 2012.

[61] S. Zheng, B. Chen, S. Ma et al., "Experimental study on the regulating effect of moxibustion on knee osteoarthritis in rabbits," Journal of Beijing University of Traditional Chinese Medicine, vol. 31, no. 5, pp. 358-360, 2008.

[62] J. He, M. S. Cortina, and H. E. P. Bazan, "The PEDF neuroprotective domain plus DHA induces corneal nerve regeneration after experimental surgery," Investigative Opthalmology \& Visual Science, vol. 56, no. 6, pp. 3505-3513, 2015. 


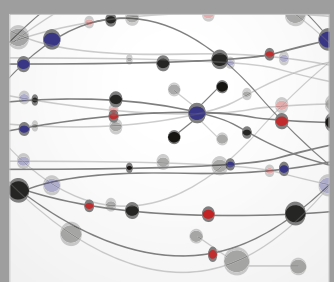

The Scientific World Journal
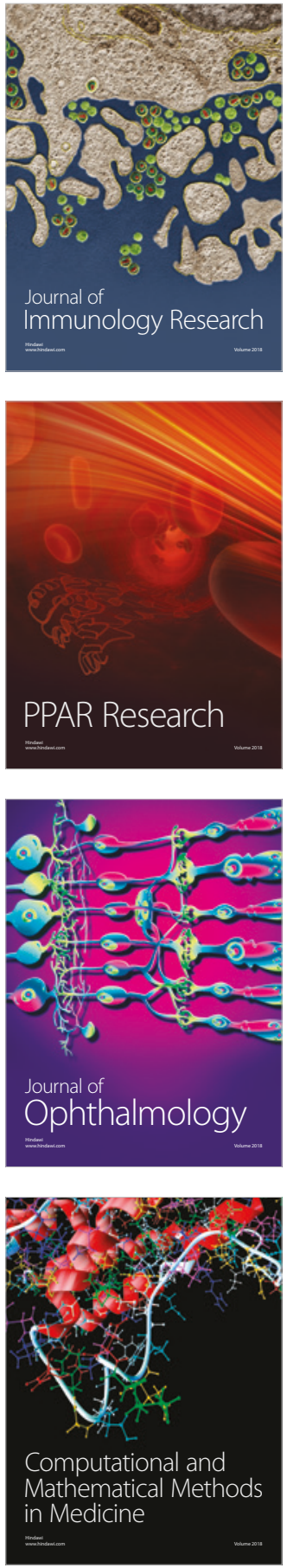

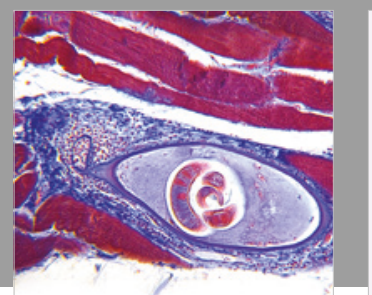

Gastroenterology Research and Practice

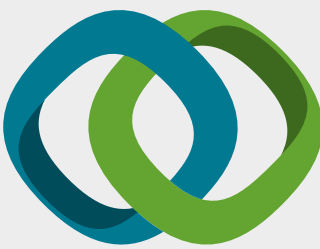

\section{Hindawi}

Submit your manuscripts at

www.hindawi.com
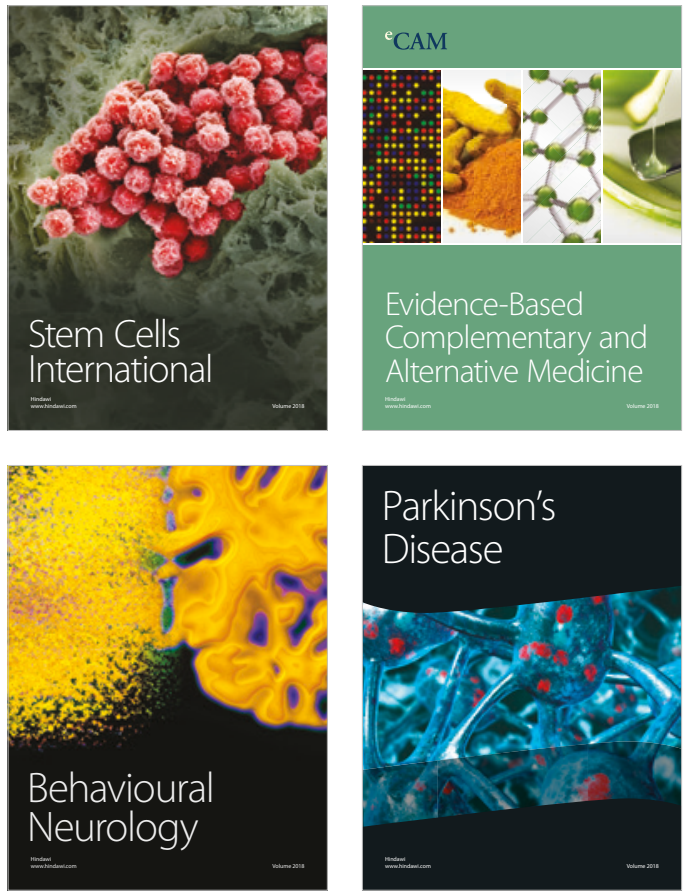

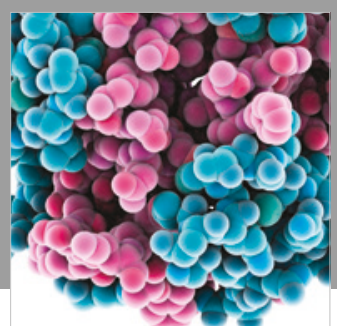

ournal of

Diabetes Research

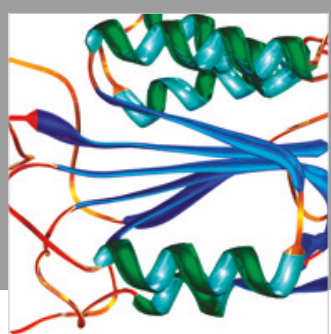

Disease Markers
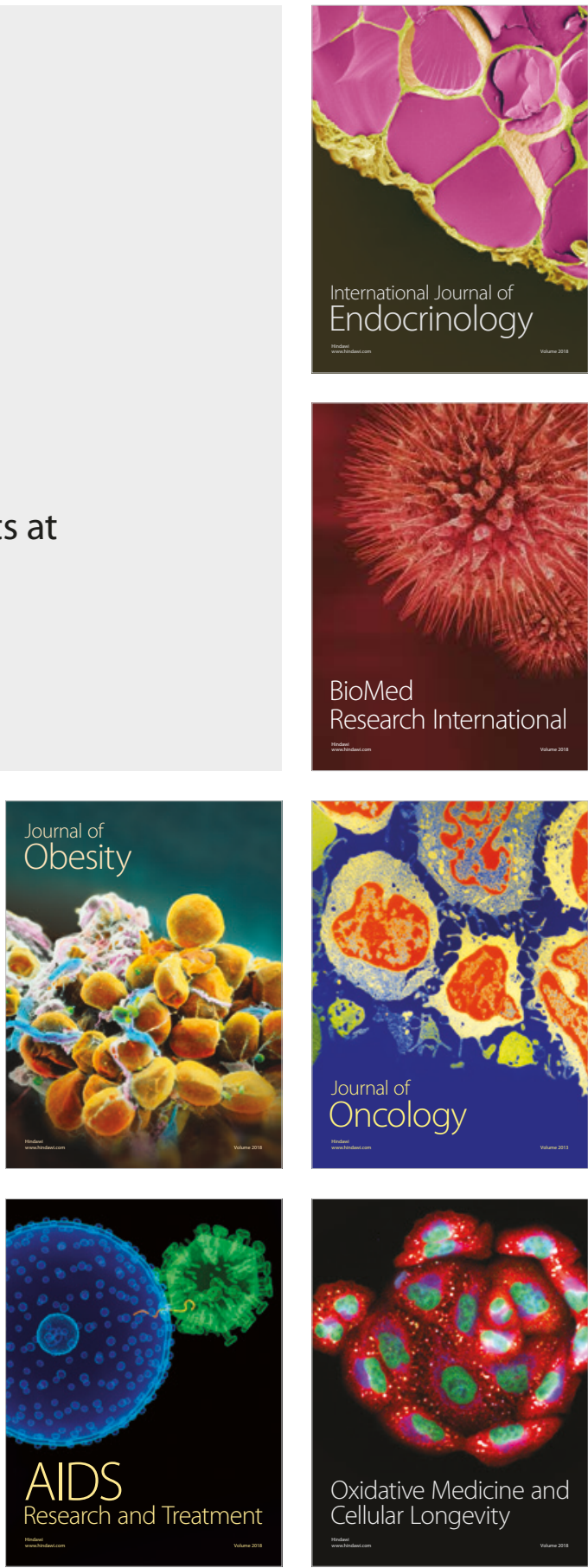